\title{
3 Research Square

\section{NK cells infiltrating in the central nervous system aggravate brain injury of mice caused by Angiostrongylus cantonensis infection}

\section{Rong Zhang}

Nanjing Medical University

Tingting Miao

Nanjing Medical University

Min Qin

Nanjing Medical University

Chengsi Zhao

Nanjing Medical University

Wei Wang

Nanjing Medical University

Chengcheng Zhang

Nanjing Medical University

Xinjian Liu

Nanjing Medical University

\section{Ying Chen}

The Affiliated Wuxi Maternity and Child Health Care Hospital of Nanjing Medical University

\section{Ailing Chen}

The Affiliated Wuxi Maternity and Child Health Care Hospital of Nanjing Medical University

Yong Wang ( $\nabla$ yongwsh@njmu.edu.cn )

Nanjing Medical University https://orcid.org/0000-0001-6673-8591

Research

Keywords: NK cells, Angiostrongylus cantonensis, infection, brain injury, central nervous system

Posted Date: December 31st, 2020

DOl: https://doi.org/10.21203/rs.3.rs-136369/v1

License: (c) (i) This work is licensed under a Creative Commons Attribution 4.0 International License.

Read Full License 


\section{Abstract}

Background Angiostrongylus cantonensis (A. cantonensis), is a food-borne zoonotic parasite that can cause central nervous system (CNS) injury characterized by eosinophilic meningitis. However, the pathogenesis of the neurological impairments caused by $A$. cantonensis infection has not been well elucidated. Natural killer cells (NK cells) are unique innate lymphocytes important in early defense against pathogens. It was reported that NK cells could migrate to the CNS after brain injury. The aim of the present study was to investigate the role of NK cells in brain injury caused by $A$. cantonensis infection.

Methods Mouse model of $A$. cantonensis infection was established by intragastric administration of third-stage larvae. Neurological impairments were evaluated by Longa's score, Clark's general score and Clark's focal score. Histopathological changes were observed by hematoxylin and eosin staining. The expression of cytokines at gene and protein levels was analyzed by PCR and ELISA, respectively. Infiltration of NK cells in the CNS was detected by immunohistochemistry and flow cytometry. Depletion of NK cells in infected mice was caused by tail vein injection of anti-asialo GM1 rabbit serum, and adoptive transfer of NK cells was performed by tail vein injection of purified splenic NK cells. NK cellmediated cytotoxicity against YAC-1 cells was detected by LDH release assay. The cytokine production ability was determined by intracellular flow cytometry and ELISA.

Results Mice developed brain inflammation and neurological impairment after $A$. cantonensis infection. The infiltration of NK cells in the CNS of $A$. cantonensis-infected mice was observed on $14 \mathrm{dpi}$ and reached the peak on $22 \mathrm{dpi}$. Compared with the normal splenic NK cells, the CNS-infiltrated NK cells of infected mice expressed lower levels of CD69, NKp46 and NKG2D, but higher levels of NKG2A, and showed enhanced cytotoxicity and increased IFN-y and TNF-a production ability. Depletion of NK cells alleviated brain injury, whereas adoptive transfer of NK cells exacerbated brain damage in A. cantonensisinfected mice.

Conclusions Our results demonstrate that NK cells infiltrate into the CNS and aggravate the brain damage after $A$. cantonensis infection. The findings improve the understanding the pathogenesis of angiostrongyliasis and expand the therapeutic intervention in CNS disease.

\section{Background}

Angiostrongylus cantonensis ( $A$. cantonensis), is a food-borne zoonotic parasite that can cause damage to the central nervous system (CNS) [1]. It was first discovered in the pulmonary arteries and hearts of domestic rats in Guangzhou, China, by Chen in 1935 [2]. So far, nearly 3000 cases of human angiostrongyliasis have been documented worldwide [3]. With the development of global logistics transportation and climate warming, $A$. cantonensis has spread from its traditional endemic regions of Southeast Asia and the Pacific islands to the American continent, Europe, Africa and Australia [4-9]. Therefore, angiostrongyliasis has become a potentially fatal globally emerging infectious disease. 
Humans and mouse are both non-permissive hosts of $A$. cantonensis and become infected via ingestion of raw or undercooked intermediate hosts including snails or slugs, or vegetables or water contaminated by the infective third-stage larvae [3]. After penetrating the intestinal wall, the larvae migrate in the body with the flow of blood, and finally settle in the CNS. These larvae in non-permissive hosts cannot develop into adults, but can survive in the form of larvae for a long time [10]. Most patients when infected with $A$. cantonensis develop eosinophilic meningitis and common clinical symptoms include headache, fever, neck stiffness, paresthesia and vomiting [11]. However, the pathogenesis of angiostrongyliasis is not fully understood. Mechanical damage to the CNS caused by the larvae' movements, inflammation and immune response caused by the larval secretion and excreta, may be involved [11-13].

Natural killer cells (NK cells) are a type of cytotoxic lymphocytes critical to the innate immune system [14]. NK cells lack T and B cell receptors and undergo activation without antigen presentation. They are able to rapidly kill target cells by cytotoxicity and produce an abundance of cytokines [15]. NK cells are dispersed throughout lymphoid and non-lymphoid tissues. It was reported that NK cells could migrate to the CNS under several pathological conditions, such as brain ischemia, traumatic injury, or infections [16]. However, very little is known about whether NK cells are involved in the CNS damage caused by $A$. cantonensis infection.

The objective of this study was to investigate the role of NK cells in A. cantonensis infection. Our findings will be helpful to further elucidate the pathogenesis of angiostrongyliasis and expand therapeutic intervention in CNS disease.

\section{Methods}

\section{Animal experiments}

A total of 300 female BALB/c mice (6-8 weeks old and weighing 18-20 g) were purchased from the Animal Core Facility of Nanjing Medical University (China), maintained in a specific pathogen-free environment, and provided unlimited access to food and water. All experiments were performed in strict compliance with the institutional guidelines and were approved by the Institutional Animal Care and Use Committee of Nanjing Medical University (Approval No. IACUC-1812040). Each mouse was infected with 20 A. cantonensis third-stage larvae (L3) by intragastric administration. L3 were isolated from $A$. cantonensis-infected Biomphalaria glabrata using the method described previously [13]. The mice were euthanized on 10, 14, 18, 22, and 26 days post-infection (dpi).

\section{Experimental Grouping}

The experiment was grouped according to different objectives.

To detect the effect of $A$. cantonensis infection on mice and the percentage and number of NK cells, the mice were divided into five groups according to the time of infection with three to twelve mice per group: $0,10,14,18$ and $22 \mathrm{dpi}$. 
When detecting the phenotypic and functional changes of NK cells, cells were isolated from mice on 18 dpi and divided into three groups with three to four mice per group: splenic NK cells of uninfected mice (uninfected sNK), splenic NK cells of infected mice (infected sNK) and brain NK cells of infected mice (infected bNK).

In the experiment of NK cell depletion or adoptive transfer, the mice were euthanatized on $18 \mathrm{dpi}$ and divided into four groups with three to twelve mice per group: uninfected group, infected group, NK depleted/transferred group and depleted/transferred control group.

\section{Neurological Impairment Evaluation}

Longa's score, Clark's general score and Clark's focal score were used to evaluate the neurological impairment of mice infected with $A$. cantonensis. Longa's score is based on a five-point scale, where 0 point indicates no neurologic deficit, 1 point (failure to extend forepaw fully) a mild focal neurologic deficit, 2 point (circling to one side) a moderate focal neurologic deficit, 3 point (falling to one side) a severe focal deficit, and animals with 4 point cannot walk spontaneously or lose consciousness [17]. Clark's general score includes hair, ears, eyes, posture, autonomous movement, and epileptic seizures. Clark's focal score comprises of body symmetry, gait, climbing, rotation test, forelimb symmetry and beard reactivity [18]. Clark's general score and Clark's focal score are between 0 and 28. The higher the score, the more serious the neurological impairment is. The mice were scored by two technicians using a blind method.

\section{Histopathological Examination}

Mice were perfused transcardially with $0.9 \%$ sodium chloride followed by $4 \%$ paraformaldehyde after anesthetized with $2 \%$ pentobarbital sodium (Sigma-Aldrich, USA). Brain samples were collected, fixed in $10 \%$ neutral formalin, embedded in paraffin, and cut into $3 \mu \mathrm{m}$-thick sections. Brain sections were then deparaffinized in xylene, rehydrated via graded alcohols and stained with hematoxylin and eosin (H\&E) (Biosharp, Wuhan, China). The sections were observed and photographed under a light microscope (Leica, Heidelberg, Germany).

For immunohistochemistry $(\mathrm{IHC})$ analysis, brain sections were subjected to antigen retrieval by boiling the slices in citrate buffer $\left(\mathrm{pH}\right.$ 6.0) with high heat for $15 \mathrm{~min}$. Then sections were treated with $3 \% \mathrm{H}_{2} \mathrm{O}_{2}$ for $10 \mathrm{~min}$ to remove endogenous peroxidase, blocked with $5 \%$ rabbit serum at room temperature for $20 \mathrm{~min}$, and incubated with rabbit anti-mouse CD49b monoclonal antibody (mAb) (Abcam, Cambridge, UK) at $4{ }^{\circ} \mathrm{C}$ overnight. After being washed in PBS, the sections were incubated with an HRP-conjugated secondary antibody (DAKO, Glostrup, Denmark) at room temperature for $15 \mathrm{~min}$ and then stained with 3, 3'-diaminobenzidine (DAB) for $10 \mathrm{~min}$. Haematoxylin was used for cell nuclei detection. The sections were visualized and digitally scanned with a light microscope.

\section{Quantitative Reverse Transcription PCR (QRT-PCR)}


Total RNA was extracted from mouse brains using TRIzol Reagent (Thermo Fisher Scientific, USA) and reverse-transcribed to cDNA using a PimerScript ${ }^{\text {TM }}$ RT Master Mix (TaKaRa, Kusatsu, Japan). qRT-PCR was performed on the LightCycler480® Real-Time PCR System (Roche, Reinach, Switzerland) with the RealUniversal Color PreMix (SYBR Green) (Tiangen, Beijing, China), in accordance with the manufacturer's instructions. The primer sequences were shown in Table 1. The mRNA levels of these genes were measured by the $\mathrm{Ct}$ value (threshold cycle), and the relative expression levels were calculated with the $2^{-\Delta \Delta C t}$ method.

Table 1

Primer sequences used for qRT-PCR

\begin{tabular}{|lll|}
\hline Gene & Forward primer sequence $\left(\mathbf{5}^{\prime} \rightarrow \mathbf{3}^{\prime}\right)$ & Reverse primer sequence $\left(\mathbf{5}^{\prime} \rightarrow \mathbf{3}^{\prime}\right)$ \\
\hline IL-1 $\beta$ & GCAACTGTTCCTGAACTCAACT & ATCTTTTGGGGTCCGTCAACT \\
\hline IL-6 & GAGGATACCACTCCCAACAGACC & AAGTGCATCATCGTTGTTCATACA \\
TNF-a & CTGTAGCCCACGTCGTAGC & TTGAGATCCATGCCGTTG \\
GAPDH & AGGTCGGTGTGAACGGATTTG & TGTAGACCATGTAGTTGAGGTCA \\
\hline
\end{tabular}

\section{Detection Of Cytokine Expression In Brain Tissue}

Each brain tissue was added into $4 \mathrm{~mL}$ tissue lysate (RayBiotech, USA) and $20 \mu \mathrm{L}$ protease inhibitor (Merck, Germany) and homogenized in a gentle MACS separator (MiltenyiBiotec, Bergisch Gladbach, Germany). The total protein concentration in each sample was detected by BCA Protein Assay Kit (Tiangen, Beijing, China) according to the manufacturer's instructions. And then, the levels of cytokines (IL-1 $\beta$, IL- 6 and TNF- $\alpha$ ) in brain tissue homogenate samples were determined with commercial ELISA Kits (MultiSciences, Hangzhou, China) according to the manufacturer's manuals.

\section{Cell Isolation}

Blood was collected by eyeball bleeding and gathered in tubes with $1 \%$ heparin sodium (Sigma-Aldrich, USA). The mice were anesthetized and perfused as described previously. And then, the brain, spleen, tibia, and femur were collected from the mice respectively.

Brain tissues were homogenized in grinders and filtered through a 70- $\mu \mathrm{m}$ cell strainer. And then cell pellets were resuspended in 30\% Percoll (GE Healthcare, Pittsburgh, USA) and centrifuged against $70 \%$ Percoll. The cells between the $30-70 \%$ Percoll interfaces were collected as the brain mononuclear cells. Spleen was grinded with a syringe core and filtered through a nylon membrane. Erythrocytes were lysed and removed using Red Cell Lysis Buffer (Beyotime, Shanghai, China). Blood was double diluted and layered on the Ficoll-Paque (GE Healthcare, Pittsburgh, USA). After centrifugation, peripheral blood mononuclear cells (PBMCs) were distributed between plasma and Ficoll-Paque. PBMCs were transferred and resuspended in PBS. The tibia and femur bones were used to prepare bone marrow cells. The 
medullary cavity was washed repeatedly with RPMI-1640 (Gibco B, Gaithersburg, MD, USA) by syringe and bone marrow cells were collected and separated from erythrocytes.

NK cells were purified from brain mononuclear cells and splenic lymphocytes using a magnetic cell sorting system (MACS) incorporating anti-mice CD49 MicroBeads (MiltenyiBiotec, Bergisch Gladbach, Germany), following the manufacturer's instructions. The purity of $C D 3^{-} \mathrm{CD} 49^{+} \mathrm{NK}$ cells after sorting was over $90 \%$ detected by FCM (Additional file 1: Figure S1).

\section{Flow Cytometry (FCM)}

Cells isolated from the brain, spleen, peripheral blood, and bone marrow were prepared to single cell suspension and resuspended in FCM buffer (0.5\% BSA in PBS). For Cell surface marker detection, cells were incubated with TruStain FcX'M anti- CD16/32 (Biolegend, San Diego, USA) to block Fc-receptor for 5 min at $4{ }^{\circ} \mathrm{C}$ and then stained with the following specific antibodies: anti-CD45-percp-cy5.5, anti-CD3FITC, anti-CD49b-APC, anti-CD122-PE, anti-CD69-PE, anti-NKp46-PE, anti-NKG2D-PE, anti-NKG2A-PE, antiCD107a-PE mAbs or isotype controls (Biolegend, San Diego, USA) for 30 min at $4{ }^{\circ} \mathrm{C}$. Cells were then detected on a Verse flow cytometer (BD Biosciences, San Jose, CA, USA). Data analysis was performed using FlowJo software (TreeStar, Ashland, USA).

For intracellular cytokine analysis, cells were cultured at a density of $2 \times 10^{6} / \mathrm{ml}$ densities in 12-well plates and stimulated with $2 \mu \mathrm{L} / \mathrm{mL}$ Leukocyte Activation Cocktail plus GolgiPlug (BD Biosciences, San Jose, CA, USA) for $5 \mathrm{~h}$. Cells were collected and stained with anti-CD45-percp-cy5.5, anti-CD3-FITC, anti-CD49bAPC mAbs for 30 min at $4{ }^{\circ} \mathrm{C}$. After washing, cells were fixed and permeabilized using Cytofix/Cytoperm ${ }^{\text {TM }}$ Fixation/Permeabilization Kit (BD Biosciences, San Jose, CA, USA) according to the manufacturer's instructions. And then cells were incubated with anti-TNF-a-PE, anti- IFN-y-BV421 mAbs or isotype controls (Biolegend, San Diego, USA) for $30 \mathrm{~min}$ at $4{ }^{\circ} \mathrm{C}$. Cells were detected and data were analyzed as described previously.

\section{NK Cell Cytotoxicity Assays}

NK cell-mediated cytotoxicity was determined using the Cytotoxicity Detection KitPLUS (Roche, Reinach, Switzerland) based on the measurement of LDH released from damaged cells according to the manufacturer's manual. Purified NK cells (as effector cells) were incubated with YAC-1 cells (as target cells) at various effector cell/target cell ratios (1:1, 5:1, 10:1, 20:1) in 96-well plates for $3.5 \mathrm{~h}$. All test samples were prepared in triplicate. Reaction mixture and stop solution were added into each well in turn. The absorbance of the samples was measured at $490 \mathrm{~nm}$ by an ELISA reader. The percentage of NK cellmediated cytotoxicity was calculated by ODs using the following equation: Cytotoxicity $(\%)=$ (effector/target cell mix - effector cell control - low control) / (high control - low control) $\times 100$.

\section{Detection Of NK Cells Cytokine Secretion}


Freshly purified NK cells were cultured in 24-well plates at a density of $1 \times 10^{6} / \mathrm{mL}$ in the presence of $1 \mathrm{ng} / \mathrm{mL}$ IL-12 (PeproTech, Rocky Hill, NJ, USA). After $24 \mathrm{~h}$ of incubation, the culture supernatants were harvested. The concentrations of TNF- $a$ and IFN- $y$ in supernatants were detected using Mouse TNF-a or IFN- $y$ High Sensitivity ELISA Kits (MultiSciences, Hangzhou, China) according to the instructions of the manufacturer.

\section{Depletion Of NK Cells}

5-7 Ganglio-N-tetraosylceramide (asialo GM1) is a glycolipid expressed on NK cells in mice, rats, and humans. Depletion of NK cells in vivo in infected mice was induced by tail vein injection of anti-asialo GM1 rabbit serum (Wako Pure Chemical Industries, Japan) according to the instructions from manufacturer and other researchers $[19,20]$. Injection time and dose were shown in Table 2. Normal rabbit serum (Abbkine, California, USA) were given to the infected mice at the same time as control. To evaluate the effect of NK cell depletion, the mice were euthanatized at $18 \mathrm{dpi}$ and the percentage and number of NK cells in brain and spleen were analyzed by FCM.

Table 2

Injection time and dose of anti-asialo GM1

\begin{tabular}{|lllll|}
\hline Injection & 1st & 2nd & 3rd & 4th \\
\hline Days post-infection & 0 & 5 & 10 & 15 \\
Dose $(\mu \mathrm{L}$ per mouse) & 20 & 20 & 20 & 20 \\
\hline
\end{tabular}

\section{Adoptive Transfer Of NK Cells}

The splenic NK cells from normal mice were purified using MACS and were suspended in PBS. The NK cells $\left(1 \times 10^{6} /\right.$ mouse in $\left.200 \mu \mathrm{L} \mathrm{PBS}\right)$ were transferred to the infected mice by tail vein injection on $12 \mathrm{dpi}$ according to the protocols published by other investigators [21]. The respective diluents were injected to the control mice simultaneously. To evaluate the effect of adoptive transfer, the mice were euthanatized at $18 \mathrm{dpi}$ and the percentage and number of NK cells in brain and spleen were analyzed by FCM.

\section{Statistical analysis}

Statistical analyses were performed using GraphPad Prism 5.0 (GraphPad Software, San Diego, USA). Survival curve comparison was determined using Log-rank Test. Comparison of the neurological impairment scores was performed using non-parametric test. The difference between two groups was compared using independent-samples $T$ test. Multiple comparison procedures were carried out with oneway analysis of variance (ANOVA). The correlation between the percentage and number of NK cells in different tissues was analyzed by linear correlation. The data are presented as the mean \pm standard deviation (SD). A $P$ value $<0.05$ was considered statistically significant. 


\section{Results}

\section{A. cantonensis infection causes neurological impairment and brain inflammation in mice}

We constructed a mouse model of $A$. cantonensis infection by intragastric administration of third-stage larvae. To evaluate the pathological damage caused by $A$. cantonensis infection, the survival rate, body weight, neurological function, histopathology, and expression of cytokines in brain tissue were detected on $0,10,14,18$ and 22 dpi.

The survival rate of mice decreased gradually with the extension of infection time (Fig. 1a). Only $50 \%$ of the mice survived on $22 \mathrm{dpi}$ and the survival rate of mice was merely $8.33 \%$ on $30 \mathrm{dpi}$. The weight of infected mice decreased significantly compared with that of uninfected mice on $18 \mathrm{dpi}$ and $22 \mathrm{dpi}$ (Fig. 1b).

After infection, various symptoms of neurologic deficit appeared in mice including erect hair, arched back, falling to one side when walking, and circling to one side continuously when their tails were lifted. Some mice even could not walk spontaneously, became blind or lost consciousness (Fig. 1c). The Longa's score, Clark's general score and Clark's focal score used to evaluate neurological impairment elevated significantly on 18 and 22 dpi compared with that of 0 dpi (Fig. 1 d-f).

Then we observed the histopathological changes of brain tissue in mice. On $10 \mathrm{dpi}$, there were a few red bleeding spots in the brain tissue, and 1-2 brown, slim, and curving fourth-stage larvae(L4)appeared on the surface. The increased hemorrhage and more invasive larvae were found in the brain tissue from 14 to $22 \mathrm{dpi}$ (Fig. 1g). And then, we prepared the brain tissue sections with H\&E staining. On $14 \mathrm{dpi}$, the meninges became thicker and a few inflammatory cells infiltrated under the meninges. On $18 \mathrm{dpi}$, the number of infiltrating inflammatory cells increased. On $22 \mathrm{dpi}$, the meninges were damaged, a large number of inflammatory cells infiltrated under the meninges, and multiple areas of red bleeding area were found (Fig. 1h).

We further examined the gene and protein levels of various inflammatory cytokines in brain tissue. The mRNA levels of IL-1 $\beta$, IL-6, TNF- $\alpha$ and the protein levels of IL- 6 increased significantly on 14, 18 and 22 $\mathrm{dpi}$, and the protein levels of IL-1 $\beta$ and TNF-a elevated significantly on 14 and $18 \mathrm{dpi}$, compared with that on 0 dpi (Fig. 1i-n).

\section{NK cells infiltrate into the CNS of mice infected with $A$. cantonensis}

H\&E staining showed that many inflammatory cells infiltrated into the CNS after $A$. cantonensis infection. In order to detect the presence of NK cells in these inflammatory cells, we performed IHC staining on brain tissue sections using anti-CD49b mAb as NK cell marker. Figure $2 a$, b showed that no NK cell was observed in the CNS on $0 \mathrm{dpi}$ and $10 \mathrm{dpi}$. On $14 \mathrm{dpi}$, a small number of NK cells stained brown appeared under the meninges indicating that NK cells began to infiltrate into the CNS. On $18 \mathrm{dpi}$, more NK cells appeared in the CNS and the number of CNS-infiltrated NK cells peaked on $22 \mathrm{dpi}$. 
To monitor NK cells quantitatively, brain mononuclear cells were isolated and analyzed by FCM at various time points. As shown in Fig. 2c-e, almost no NK cells were detected in the brain tissue at 0 dpi and 10 dpi. NK cells appeared in the CNS from $14 \mathrm{dpi}$ and the percentage and number of CNS-infiltrated NK cells increased gradually with the extension of infection time. The highest percentage of CNS-infiltrated NK cells appeared on $22 \mathrm{dpi}$ ( $22 \mathrm{dpi}$ vs $0 \mathrm{dpi}$ : $17.47 \pm 6.11 \%$ vs $0.45 \pm 0.12 \%, P<0.001$ ) and the maximum number of CNS-infiltrated NK cells were found on $18 \mathrm{dpi}\left(18 \mathrm{dpi}\right.$ vs $0 \mathrm{dpi}: 1.92 \pm 0.43 \times 10^{5}$ vs $696.9 \pm$ 617.2, $P<0.001)$.

To elucidate the origin of NK cells in the CNS, we further analyzed the percentage and number of NK cells in the splenic lymphocytes, peripheral blood mononuclear cells and bone marrow cells by FCM. The results showed that the percentage and number of NK cells in splenic lymphocytes decreased on 14, 18 and 22 dpi. (Fig. 3a, d, e). So did the number of NK cells in PBMCs from 10 dpi to 22 dpi (Fig. 3b, e, f). Furthermore, the percentage and number of sNK (NK cells in spleen) were negatively correlated with those of bNK (NK cells in brain) $(r=0.79, P<0.01 ; r=0.85, P<0.01)$ (Fig. 3g, $h)$. The percentage and number of pbNK (NK cells in PBMCs) and bNK were also negatively correlated, but there was no statistical significance (Fig. 3i, j).

However, the percentage of total NK cells (bmNK, CD122+), NK precursor cells (NKP, CD 49b ${ }^{-} \mathrm{CD} 122^{+}$) and mature NK cells (mature NK, CD49b ${ }^{+} C D 122^{+}$) in bone marrow all significantly increased on 18 and 22 dpi. (Fig. 4a-d). And the ratio of NKP to mature NK in bone marrow up-regulated (Fig. 4e). In addition, the percentage of total NK cells in bone marrow was positively correlated with that of bNK $(r=0.87, P<0.001)$ (Fig. 4f).

\section{CNS-infiltrated NK cells of $A$. cantonensisinfected mice have elevated cytotoxicity and secretory ability}

We further detected the phenotypic and functional changes of NK cells after $A$. cantonensis infection. We measured the expression of activation marker CD69, activated receptor NKp46 and NKG2D, and inhibitory receptor NKG2A on NK cells by FCM. Figure 5a-g revealed that infected bNK expressed lower levels of CD69, NKp46 and NKG2D, but higher levels of NKG2A, and infected sNK expressed lower levels of CD69, NKp46, but higher levels of NKG2A, compared with uninfected sNK. Although the phenotypic changes of infected bNK were greater than those of infected SNK, there was no statistical difference between them.

Then we detected NK cell-mediated cytotoxicity against YAC-1 cells by LDH release assay. As shown in Fig. 6a, infected bNK and infected sNK had enhanced cytotoxicity, compared with uninfected sNK (Effect cells: Target cells $=20: 1,19.37 \%$ vs $10.59 \%$ vs $8.35 \%$ ). Furthermore, the expression of CD107a, a surface marker of NK cell degranulation, on infected bNK and infected sNK was up-regulated compared with that of uninfected sNK (Fig. 6b, c).

The ability of NK cells to secrete cytokines was determined by two methods: ELISA (secretory levels) and FCM (intracellular levels). Firstly, we detected the concentrations of TNF-a and IFN- $y$ in the culture supernatant of NK cells after IL-12 stimulation using ELISA. The levels of TNF- $\alpha$ and IFN- $\gamma$ secreted by infected bNK and infected sNK were significantly higher than those of uninfected sNK (Fig. 6d). 
Subsequently, purified NK cells were stimulated and measured by FCM. The percentage of IFN- $\gamma^{+}$cells in infected bNK was higher than that of uninfected sNK, while the percentage of TNF- $\mathrm{a}^{+}$or IFN- ${ }^{+}$cells in infected sNK did not change significantly (Fig. 6e, f).

\section{NK cells aggravate brain injury of mice caused by $A$. cantonensis infection}

We designed NK cell depletion and adoptive transfer experiments to elucidate the role of NK cells in brain injury caused by $A$. cantonensis infection.

Firstly, NK cells were depleted by tail vein injection of anti-asialo GM1 serum to infected mice. The percentage and number of NK cells in brain and spleen of infected mice significantly decreased after NK cell depletion (Fig. 7a-d). The survival rate of NK-depleted mice increased $(P<0.01)$ (Fig. 7e), body weight elevated $(17.35 \pm 1.38 \mathrm{~g}$ vs $15.61 \pm 1.1 \mathrm{~g}, P<0.05)$ (Fig. 7f), neurological impairment score decreased slightly (Fig. 7g-i), brain inflammation alleviated (Fig. 7j), and the levels of inflammatory cytokines in brain tissue reduced(IL-1 $\beta 25.88 \pm 2.38 \mathrm{pg} / \mathrm{mg}$ vs $30.75 \pm 2.83 \mathrm{pg} / \mathrm{mg}$, IL-6 $12.88 \pm 1.23 \mathrm{pg} / \mathrm{mg}$ vs $15.08 \pm$ $1.13 \mathrm{pg} / \mathrm{mg}$, TNF-a $57.17 \pm 4.20 \mathrm{pg} / \mathrm{mg}$ vs $64.68 \pm 4.34 \mathrm{pg} / \mathrm{mg}, P<0.05)$ (Fig. $7 \mathrm{k}-\mathrm{p})$, compared with the infected mice on 18dpi. These results showed that depletion of NK cells alleviated brain injury in $A$. cantonensis-infected mice.

And then purified splenic NK cells were transferred to infected mice by tail vein injection. The percentage of NK cells in brain and spleen of infected mice increased significantly after adoptive transferring NK cells (Fig. 8a-d). Compared with the infected mice, the survival rate, body weight and neurological impairment score of NK-transferred mice did not change significantly (Fig. 8e-i), but brain inflammation aggravated (Fig. 8j), and the concentrations of inflammatory cytokines in brain tissue elevated (IL-1 $\beta$ $36.36 \pm 1.89 \mathrm{pg} / \mathrm{mg}$ vs $30.75 \pm 2.83 \mathrm{pg} / \mathrm{mg}, P<0.05 ; \mathrm{IL}-618.08 \pm 0.55 \mathrm{pg} / \mathrm{mg}$ vs $15.08 \pm 1.13 \mathrm{pg} / \mathrm{mg}, P<$ 0.01 ; TNF-a $76.84 \pm 1.21 \mathrm{pg} / \mathrm{mg}$ vs $64.68 \pm 4.34 \mathrm{pg} / \mathrm{mg}, P<0.01$ ) (Fig. 8k-p) on $18 \mathrm{dpi}$. It was suggested that adoptive transfer of NK cells exacerbated brain damage in mice caused by $A$. cantonensis.

\section{Discussion}

Angiostrongylosis, a food-borne parasitic disease, is caused by the larvae of $A$. cantonensis in the host's central nervous system [22]. NK cells are important innate immune effector cells. They can be swiftly mobilized by danger signals and are among the earliest arrivals at target organs against pathogen infection [23]. However, the role of NK cells in the CNS damage caused by A. cantonensis infection remains elusive. Our previous work has reported that NK cells in the spleen and peripheral blood showed quantitative reduction and functional changes in an A. cantonensis-infected mice model [24]. In the current study, we focused on the NK cells in the CNS. We found a large number of NK cells infiltrated into the CNS of mice after $A$. cantonensis infection and these CNS-infiltrated NK cells had elevated cytotoxicity and secretory ability. Moreover, we demonstrated that NK cells aggravated brain injury of mice caused by $A$. cantonensis infection. 
Mice and humans are both non-permissive hosts of $A$. cantonensis, and the pathogenic process is comparatively similar [25]. Consistent with other reports [26-28], we successfully constructed a mouse model infected with $A$. cantonensis and observed serious neurological damage from $18 \mathrm{dpi}$ to $22 \mathrm{dpi}$. What is noteworthy in this study is that we confirmed that NK cells infiltrated into the CNS after $A$. cantonensis infection. The results of $\mathrm{IHC}$ and FCM showed that NK cells began to appear in the brain tissues at $14 \mathrm{dpi}$. The percentage and number of NK cells increased gradually with the extension of infection time until $22 \mathrm{dpi}$. The CNS, including the brain and spinal cord, is considered as an immune privileged organ because of the low permeability of the brain-blood barrier (BBB). However, it is conceivable that peripherally activated lymphocytes, including NK cells, might also be able to penetrate the BBB and infiltrate into the CNS under some pathological conditions. In human ischemic brain tissue and a permanent middle cerebral artery occlusion (pMCAO) mouse model, infiltration of NK cells into the ischemic infarct region are observed [29-31]. NK cells are activated in the periphery and then migrated into the CNS of experimental autoimmune encephalomyelitis (EAE) mice [32]. NK cells can be detected in mouse CNS tissues during a variety of infections, including Semliki Forest virus (SFV) [33], murine coronavirus [34] and L. monocytogenes [35]. NK cells are also recruited to the CNS in glioma-bearing mice and constitute approximately $50 \%$ of all leukocytes in the CNS [36].

To explore the origin of NK cells in the CNS, we examined the distribution of NK cells in A. cantonensisinfected mice. The results showed that the percentage and number of NK cells in spleen and in peripheral blood both decreased, consistent with our previous study [24], while the percentage of NK cells in bone marrow increased after $A$. cantonensis infection. In addition, the percentage and number of splenic NK cells were negatively correlated with those of brain NK cells, while the percentage of NK cells in bone marrow was positively correlated with brain NK cells. It was suggested that the CNS-infiltrated NK cells probably were related with the increased hematopoiesis of bone marrow and migration of peripheral NK cells after $A$. cantonensis infection. NK cells originate from bone marrow, are mainly distributed in peripheral blood and spleen, and some lymphatic tissues [15]. However, the distribution of NK cells is not static because these cells can recirculate between organs. NK cells can respond to a large array of chemokines and be recruited to distinct sites in several pathological circumstances [16]. The detailed trafficking patterns of NK cells are not very well characterized. Nevertheless, it appears that chemokines produced by cells that are unique to specific organs may have a role in orchestrating NK cell migration to each organ [16]. It was reported that NK cells might be recruited to the CNS by chemokines such as $\mathrm{CX}_{3} \mathrm{CL} 1$ produced by neurons [37] and CCL2 and CXCL10 produced by microglia, astrocytes or infiltrating inflammatory cells $[29,38]$. The mechanism of recruitment of NK cells to the CNS after $A$. cantonensis infection needs to be further studied.

NK cells are a kind of special lymphocytes, which can lyse the target cells directly and produce various cytokines to regulate immune response [39]. To further identify the characteristics of the CNS-infiltrated NK cells, we detected the phenotype and function of NK cells after $A$. cantonensis infection. The results showed that the expression of activation molecule CD69, activating receptor NKp46 and NKG2D on the CNS-infiltrated NK cells of infected mice was decreased compared with the splenic NK cells of uninfected

Page $11 / 29$ 
mice, while the expression of inhibitory receptor NKG2A increased. Consistent phenotypic changes were observed in the splenic NK cells of infected mice except for NKG2D. CD69 is one of the early activation markers on NK cells and is involved in cell proliferation and signal transduction. Meanwhile, CD69 a novel regulator of the immune response, which can inhibit the cytotoxicity of NK cells by inducing the production of TGF- $\beta$. It was demonstrated that the administration of anti-CD69 mAbs can activate resting NK cells, resulting in a substantial increase in both NK-cell cytolytic activity and IFN-Y production [40]. NK cells express an array of inhibitory and activating receptors recognizing self-ligands or microbial molecules on infected and tumor cells. Coordinated acquisition of the signals originating from inhibitory and activating receptors regulates the effector functions of NK cells. The down-regulation of CD69 and activating receptors and up-regulation of inhibitory receptors on NK cells following $A$. cantonensis infection might imply the changes of their effector functions.

NK cells have a variety of biological functions, with the most important role being cytotoxicity. NK cells can directly lyse malignant cells or pathogen infected cells by several different mechanisms, including perforin/granzyme, Fas/FasL, TNF-a/TNFR-I and other pathways [15]. In our study, we found that the CNS-infiltrated NK cells of infected mice had an enhanced cytotoxicity against YAC-1 cells with higher expression of CD107a. CD107a, also known as Lysosome associated membrane protein-1 (LAMP-1), is a marker for degranulation of NK cells and $\mathrm{CD}^{+} \mathrm{T}$ cells [41]. It is reported that CD107a expression correlates with NK cell-mediated lysis of target cells and is a sensitive marker of NK cell activity [42]. NK cells can also produce a variety of cytokines in response to activation signaling to mediate immunomodulatory effects. Our results showed that the CNS-infiltrated NK cells of infected mice produced higher levels of TNF- $a$ and IFN- $y$ than splenic NK cells of uninfected mice. TNF-a is a proinflammatory cytokine that is not only cytotoxic to tumor cells but also involved in mediating antipathogen immune responses. IFN- $\gamma$ is an important immunomodulator with the function of activating macrophages, promoting $\mathrm{MHC}$ molecular expression and antigen presentation, and plays an important role in defending viral, bacterial and parasitic infections $[43,44]$. Taken together, the CNS-infiltrated NK cells in A. cantonensis-infected mice showed stronger activity with enhanced cytotoxicity and elevated production of TNF- $a$ and IFN- $\gamma$. There are no NK cells in the steady-state CNS, but NK cells might migrate into the CNS under certain pathological conditions such as $A$. cantonensis infection. After homing to the inflamed CNS, NK cells become receptive to an array of cellular components that they have not encountered in the periphery. These include astrocytes, microglia, neurons, and eosinophils, which release numerous soluble factors with diversified and perhaps coordinated effects on NK cells [16]. The exact cellular and molecular interactions that shape the phenotype and function of NK cells in the CNS still need to be determined.

We then investigated the role of NK cells in the brain damage caused by A. cantonensis infection using NK cell depletion and adoptive transfer experiments. The changes in NK cell percentage and number confirmed that NK cell depletion and adoptive transfer were effective. Our results showed that the survival rate and body weight increased, nerve injury and brain inflammation decreased after NK depletion in $A$. cantonensis-infected mice. On the contrary, the inflammation in brain aggravated after adoptive transfer 
of NK cells. We speculated that the CNS-infiltrated NK cells might play a role in exacerbating brain injury after $A$. cantonensis infection. Many studies have reported that NK cells can rapidly accumulated into the CNS under pathological conditions. However, the role of NK cells in brain injury diseases remains complex and paradoxical. Zhiguo Li [31] identified NK cells infiltrates into the CNS during early stages of intracerebral hemorrhage $(\mathrm{ICH})$. These infiltrating NK cells exacerbate BBB disruption and brain edema via cytotoxicity toward cerebral endothelial cells and recruitment of neutrophils. Gan Y [30] reported NK cells mediate exacerbation of brain infarction after ischemia via the disruption of NK cell tolerance, augmenting local inflammation and neuronal hyperactivity. Alsharifi M [33] found that NK cells exert both disease-exacerbating and protective effects in Semliki Forest virus infection in the CNS of mice. The cytolytic activity of NK cells is detrimental, while IFN-y production is beneficial for recovery from SFV infection. However, Hao J [32] demonstrated that the CNS-resident NK cells have a protective role in the brain of EAE mice, as they inhibit the activation of autoimmune T cells through the killing of activated microglia. Jiang W [45] disclosed that acetylcholine-producing NK cells attenuate CNS inflammation of EAE model via modulation of infiltrating monocytes/macrophages. NK cells play different roles in brain injury, which may be related to the initial factors of the primary disease, the time of immune response, and the overall inflammatory process [30]. After brain injury, brain-intrinsic cells, including microglia, astrocytes and neurons, secrete various chemokines which recruiting a series of inflammatory cells. As an important type of immune cell, peripheral resident NK cells are activated and migrate to brain tissue [16]. NK cells infiltrating into the CNS can build a bridge between immune system and nervous system, which not only produce cytotoxicity or cytolysis to pathogens, but also interact with other cells to affect the progress of brain injury. In the process of $A$. cantonensis infection, NK cells infiltrating in brain tissue have stronger killing function and secrete more IFN- $y$ and TNF-a. On the one hand, these CNS-infiltrated NK cells were beneficial to clearance of pathogens, on the other hand, they were also involved in the death of neural cells and aggravated brain damage.

Currently, the treatment for angiostrongyliasis includes supportive treatment and corticosteroid therapy [46]. The use of anthelmintic drugs, such as albendazole and mebendazole, to kill worms remains controversial. Because the dead worm lysis in the CNS might cause severe inflammatory response and further damage [10]. Meanwhile, Patients under the treatment of high-dose corticosteroids would experience immune suppression [47]. Therefore, it is urgent to develop new therapeutic interventions for angiostrongyliasis. We hypothesized that selective reduction of NK cell infiltration into the CNS or control

their activation may help to alleviate the brain injury caused by $A$. cantonensis infection, which need to be further investigated in our future work.

\section{Conclusion}

In summary, our study demonstrates that NK cells infiltrate into the CNS of $A$. cantonensis-infected mice. These CNS-infiltrated NK cells display enhanced cytotoxicity and secretary ability, which may contribute to aggravate brain damage. Our findings not only enrich the understanding of $A$. cantonensis pathogenesis but also provide a clue to novel potential therapeutic strategies against CNS disease. 


\section{Abbreviations}

A.cantonensis. Angiostrongylus cantonensis; BBB: Brain-blood Barrier; bNK: NK cells in brain; bmNK: NK cells in bone marrow; CNS: Central nervous system; DAB: diaminobenzidine; EAE: Experimental autoimmune encephalomyelitis; H\&E: Hematoxylin and eosin staining; ICH: intracerebral hemorrhage; IHC: Immunohistochemistry; L3: Third-stage larvae; LAMP-1: Lysosome associated membrane protein-1; MACS: Magnetic cell sorting system; NK cells: Natural killer cells; NKP: NK precursor cells; pbNK: NK cells in peripheral blood; PBMCs: Peripheral blood mononuclear cells; pMCAO: permanent middle cerebral artery occlusion; SFV; Semliki Forest virus; sNK; NK cells in spleen.

\section{Declarations}

\section{Acknowledgements}

We thank Professor Xi Sun, Sun Yat-sen University, Guangzhou, China, for providing us with the $A$. cantonensis-infected snails. We thank Associate Professor Haiwei Wu, Brown University, Center for International Health Research, Rhode Island Hospital, USA, for her advice on writing the manuscript.

\section{Authors' contributions}

ALC conceived the project and designed the experiments. YW designed the experiments, supervised the project, and was involved in all aspects of the submission. RZ performed most of the experiments, analyzed data, and wrote the manuscript. TTM was responsible for the infection and feeding of animals, performed cell isolation and detection by FCM. MQ, CSZ performed detection of cytokine by qRT-PCR and ELISA. CCZ and WW participated in neurological impairment evaluation and NK cell cytotoxicity assays. XJL and YC performed histopathological examination and data analysis. All authors read and approved the final manuscript.

\section{Funding}

This work was supported by the National Natural Science Foundation of China (No. 81501371), the Post doctorate Foundation of China (2019M651963), the Post doctorate Foundation of Jiangsu Province(2018Z093), Jiangsu Provincial Medical Youth Talent of the Project of Invigorating Health Care through Science, Technology and Education (QNRC2016165), and the Foundation of top notch young and middle-aged medical and health talents in Wuxi (BJ2020079). This work was also supported,in part, by the National Basic Research Program of China (973 Program) (No. 2010CB530004). Funders did not have a role in the design and conduct of the study; collection, management, analysis, and interpretation of the data; preparation, review, or approval of the manuscript; or decision to submit the manuscript for publication.

\section{Availability of data and materials}


The datasets used and analyzed during the current study are available from the corresponding author on reasonable request.

\section{Ethics approval and consent to participate}

All experiments were performed in strict compliance with the institutional guidelines and were approved by the Institutional Animal Care and Use Committee of Nanjing Medical University (Approval No. IACUC1812040).

\section{Consent for publication}

Not applicable.

\section{Competing interests}

The authors declare that they have no competing interests.

\section{Author details}

${ }^{1}$ Experimental Teaching Center of Basic Medicine, Nanjing Medical University, 140 Hanzhong Road, Nanjing, Jiangsu 210029, China. ${ }^{2}$ Department of Pathogen Biology, Key Laboratory of Pathogen Biology of Jiangsu Province, Nanjing Medical University, 140 Hanzhong Road, Nanjing, Jiangsu 210029, China.

${ }^{3}$ Translational Medicine Laboratory $₫$ Research Institute for Reproductive Health and Genetic Diseases, The Affiliated Wuxi Maternity and Child Health Care Hospital of Nanjing Medical University, Wuxi, Jiangsu 214002, China.

\section{References}

1. Wang QP, Wu ZD, Wei J, Owen RL, Lun ZR. Human Angiostrongylus cantonensis: an update. Eur J Clin Microbiol Infect Dis. 2012;31:389-95.

2. Chen HT. Un nouveau nématode pulmonaire, Pulmonema cantonensis, n. g., n. sp. Ann. Parasitol. Hum. Comp. 1935;13:312-7.

3. Barratt J, Chan D, Sandaradura I, Malik R, Spielman D, Lee R, et al. Angiostrongylus cantonensis: a review of its distribution, molecular biology and clinical significance as a human pathogen. Parasitology. 2016;143:1087-118.

4. Liu EW, Schwartz BS, Hysmith ND, DeVincenzo JP, Larson DT, Maves RC, et al. Rat Lungworm Infection Associated with Central Nervous System Disease - Eight U.S. States, January 2011-January 2017. MMWR. Morbidity and mortality weekly report. 2018;67:825-8.

5. Rael RC, Peterson AC, Ghersi-Chavez B, Riegel C, Lesen AE, Blum MJ. Rat Lungworm Infection in Rodents across Post-Katrina New Orleans, Louisiana, USA. Emerg Infect Dis. 2018;24:2176-83.

6. Gelis S, Spratt DM, Raidal SR. Neuroangiostrongyliasis and other parasites in tawny frogmouths (Podargus strigoides) in south-eastern Queensland. Aust Vet J. 2011;89:47-50. 
7. Iwanowicz DD, Sanders LR, Schill WB, Xayavong MV, da Silva AJ, Qvarnstrom Y, et al. Spread of the Rat Lungworm (Angiostrongylus cantonensis) in Giant African Land Snails (Lissachatina fulica) in Florida, USA. J Wildl Dis. 2015;51:749-53.

8. Federspiel F, Skovmand S, Skarphedinsson S. Eosinophilic meningitis due to Angiostrongylus cantonensis in Europe. International journal of infectious diseases : IJID : official publication of the International Society for Infectious Diseases. 2020;93:28-39.

9. Barbosa TA, Thiengo SC, Fernandez MA, Graeff-Teixeira C, Morassutti AL, Mourão FRP, et al. Infection by Angiostrongylus cantonensis in both humans and the snail Achatina (Lissachatina) fulica in the city of Macapá, in the Amazon Region of Brazil. Memorias do Instituto Oswaldo Cruz. 2020;115:e200115.

10. Lv S, Zhou XN, Andrews JR. Eosinophilic Meningitis Caused by Angiostrongylus cantonensis. ACS Chem Neurosci. 2017;8:1815-6.

11. Martins YC, Tanowitz HB, Kazacos KR. Central nervous system manifestations of Angiostrongylus cantonensis infection. Acta Trop. 2015;141:46-53.

12. Wang QP, Lai DH, Zhu XQ, Chen XG, Lun ZR. Human angiostrongyliasis. Lancet Infect Dis. 2008; 8:621-30.

13. Zhang MY, Xu YY, Pan T, Hu Y, Limpanont Y, Huang P, et al. Apoptosis and necroptosis of mouse hippocampal and parenchymal astrocytes, microglia and neurons caused by Angiostrongylus cantonensis infection. Parasit Vectors. 2017;10:611.

14. Vivier E, Raulet DH, Moretta A, Caligiuri MA, Zitvogel L, Lanier LL, et al. Innate or adaptive immunity? The example of natural killer cells. Science. 2011;331:44-9.

15. Abel AM, Yang C, Thakar MS, Malarkannan S. Natural Killer Cells: Development, Maturation, and Clinical Utilization. Frontiers in immunology. 2018;9:1869.

16. Shi FD, Ljunggren HG, Cava AL, Kaer LV. Organ-specific features of natural killer cells. Nature Reviews Immunology. 2011;11:658-71.

17. Clark WM, Lessov NS, Dixon MP, Eckenstein F. Monofilament intraluminal middle cerebral artery occlusion in the mouse. Neurol Res. 1997;19:641-8.

18. Longa EZ, Weinstein PR, Carlson S, Cummins R. Reversible middle cerebral artery occlusion without craniectomy in rats. Stroke. 1989;20:84-91.

19. Nishikado H, Mukai K, Kawano Y, Minegishi Y, Karasuyama H. NK cell-depleting anti-asialo GM1 antibody exhibits a lethal off-target effect on basophils in vivo. Journal of Immunology. 2011;186:5766-71.

20. Golic M, Haase N, Herse F, Wehner A, Vercruysse L, Pijnenborg R, et al. Natural Killer Cell Reduction and Uteroplacental Vasculopathy. Hypertension. 2016;68:964-73.

21. Voynova E, Qi CF, Scott B, Bolland S. Cutting Edge: Induction of Inflammatory Disease by Adoptive Transfer of an Atypical NK Cell Subset. Journal of Immunology. 2015;195:806-9. 
22. Martins YC, Tanowitz HB, Kazacos KR. Central nervous system manifestations of Angiostrongylus cantonensis infection. Acta tropica. 2015;141:46-53.

23. Cruzmunoz ME, Veillette A. Do NK cells always need a license to kill? Nature Immunology. 2010;11:279-80.

24. Chen AL, Qiu XY, Wang W, Zhou CL, Zeng X, Liu XJ, et al. The quantitative and functional changes of NK cells in mice infected with Angiostrongylus cantonensis. Parasitology research. 2014;113:208794.

25. Ouyang L, Wei J, Wu Z, Zeng X, Li Y, Jia Y, et al. Differences of larval development and pathological changes in permissive and nonpermissive rodent hosts for Angiostrongylus cantonensis infection. Parasitology research. 2012;111:1547-57.

26. Wang LC, Jung SM, Chen KY, Wang TY, Li CH. Temporal-spatial pathological changes in the brains of permissive and non-permissive hosts experimentally infected with Angiostrongylus cantonensis. Exp Parasitol. 2015;157:177-84.

27. Chen AL, Sun X, Wang W, Liu JF, Zeng X, Qiu JF, et al. Activation of the hypothalamic-pituitaryadrenal (HPA) axis contributes to the immunosuppression of mice infected with Angiostrongylus cantonensis. J Neuroinflammation. 2016;13:266.

28. Guo PJ, Zhan XM, Gan M, Pan ZH, Yu YJ, Zhang MC, et al. Pathological change in the brain of mice infected with Angiostrongylus cantonensis. Chinese journal of parasitology \& parasitic diseases. 2008;26:353-5.

29. Zhang Y, Gao Z, Wang D, Zhang T, Sun B, Mu L, et al. Accumulation of natural killer cells in ischemic brain tissues and the chemotactic effect of IP-10. J Neuroinflammation. 2014;11:79.

30. Gan Y, Liu Q, Wu W, Yin JX, Bai XF, Shen R, et al. Ischemic neurons recruit natural killer cells that accelerate brain infarction. Proceedings of the National Academy of Sciences of the United States of America. 2014;111:2704-9.

31. Li Z, Li M, Shi SX, Yao N, Cheng X, Guo A, et al. Brain transforms natural killer cells that exacerbate brain edema after intracerebral hemorrhage. The Journal of experimental medicine. 2020;217: e20200213.

32. Hao J, Liu R, Piao W, Zhou Q, Vollmer TL, Campagnolo DI, et al. Central nervous system (CNS)resident natural killer cells suppress Th17 responses and CNS autoimmune pathology. The Journal of experimental medicine. 2010;207:1907-21.

33. Alsharifi M, Lobigs M, Simon MM, Kersten A, Muller K, Koskinen A, et al. NK cell-mediated immunopathology during an acute viral infection of the CNS. European journal of immunology. 2006;36:887-96.

34. Hayashi T, Nagai S, Fujii H, Baba Y, Ikeda E, Kawase T, et al. Critical roles of NK and CD8 ${ }^{+}$T cells in central nervous system listeriosis. Journal of immunology. 2009;182:6360-8.

35. Trifilo MJ, Montalto-Morrison C, Stiles LN, Hurst KR, Hardison JL, Manning JE, et al. CXC chemokine ligand 10 controls viral infection in the central nervous system: evidence for a role in innate immune 
response through recruitment and activation of natural killer cells. Journal of virology. 2004;78:58594.

36. Alizadeh D, Zhang L, Brown CE, Farrukh O, Jensen MC, Badie B. Induction of anti-glioma natural killer cell response following multiple low-dose intracerebral CpG therapy. Clinical cancer research : an official journal of the American Association for Cancer Research. 2010;16:3399-408.

37. Hertwig L, Hamann I, Romero-Suarez S, Millward JM, Pietrek R, Chanvillard C, et al. CX ${ }_{3}$ CR1dependent recruitment of mature NK cells into the central nervous system contributes to control autoimmune neuroinflammation. European journal of immunology. 2016;46:1984-96.

38. Hao J, Campagnolo D, Liu R, Piao W, Shi S, Hu B, et al. Interleukin-2/ interleukin-2 antibody therapy induces target organ natural killer cells that inhibit central nervous system inflammation. Annals of neurology. 2011;69:721-34.

39. Vivier E, Tomasello E, Baratin M, Walzer T, Ugolini S. Functions of natural killer cells. Nature Immunology. 2008;9:503-10.

40. Esplugues E, Vegaramos J, Cartoixà D, Vazquez BN, Salaet I, Engel P, et al. Induction of tumor NK-cell immunity by anti-CD69 antibody therapy. Blood. 2005;105:4399-406.

41. Aktas E, Kucuksezer UC, Bilgic S, Erten G, Deniz G. Relationship between CD107a expression and cytotoxic activity. Cellular Immunology. 2009; 254:149-54.

42. Alter G, Malenfant JM, Altfeld M. CD107a as a functional marker for the identification of natural killer cell activity. Journal of Immunological Methods. 2004;294:15-22.

43. Schoenborn JR, Wilson CB. Regulation of interferon-gamma during innate and adaptive immune responses. Advances in Immunology. 2007;96:41-101.

44. Zou Y, Chen T, Han M, Wang H, Yan W, Song G, et al. Increased killing of liver NK cells by Fas/Fas ligand and NKG2D/NKG2D ligand contributes to hepatocyte necrosis in virus-induced liver failure. Journal of immunology (Baltimore, Md. : 1950). 2010;184:466-75.

45. Jiang W, Li D, Han R, Zhang C, Jin WN, Wood K, et al. Acetylcholine- producing NK cells attenuate CNS inflammation via modulation of infiltrating monocytes/macrophages. Proceedings of the National Academy of Sciences of the United States of America. 2017;114:E6202-e11.

46. Graeff-Teixeira C, Morassutti AL, Jones MK. Diagnosing and Understanding Angiostrongyliasis, A Zoonotic Cause of Meningitis. ACS Chem Neurosci. 2018;9:393-4.

47. Cowie RH. Angiostrongylus cantonensis: Agent of a Sometimes Fatal Globally Emerging Infectious Disease (Rat Lungworm Disease). Acs Chemical Neuroscience. 2017;8:2102-4.

\section{Figures}



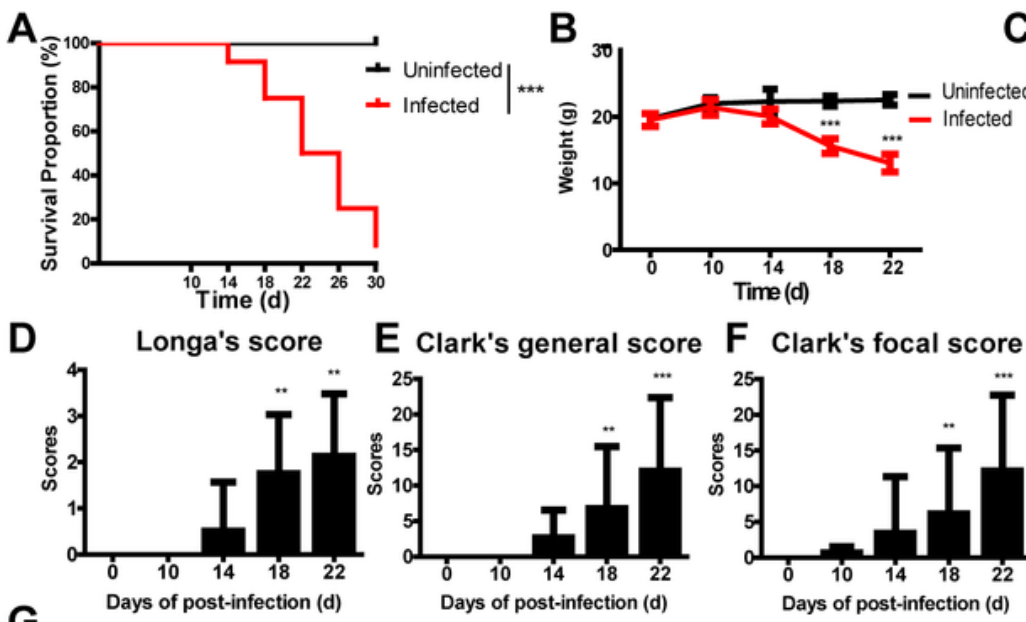

E Clark's general score $F$ Clark's focal score

$\mathbf{0} \mathbf{d}$
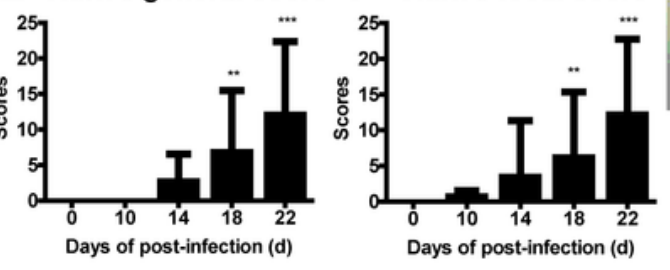

$10 \mathrm{~d}$

$14 \mathrm{~d}$
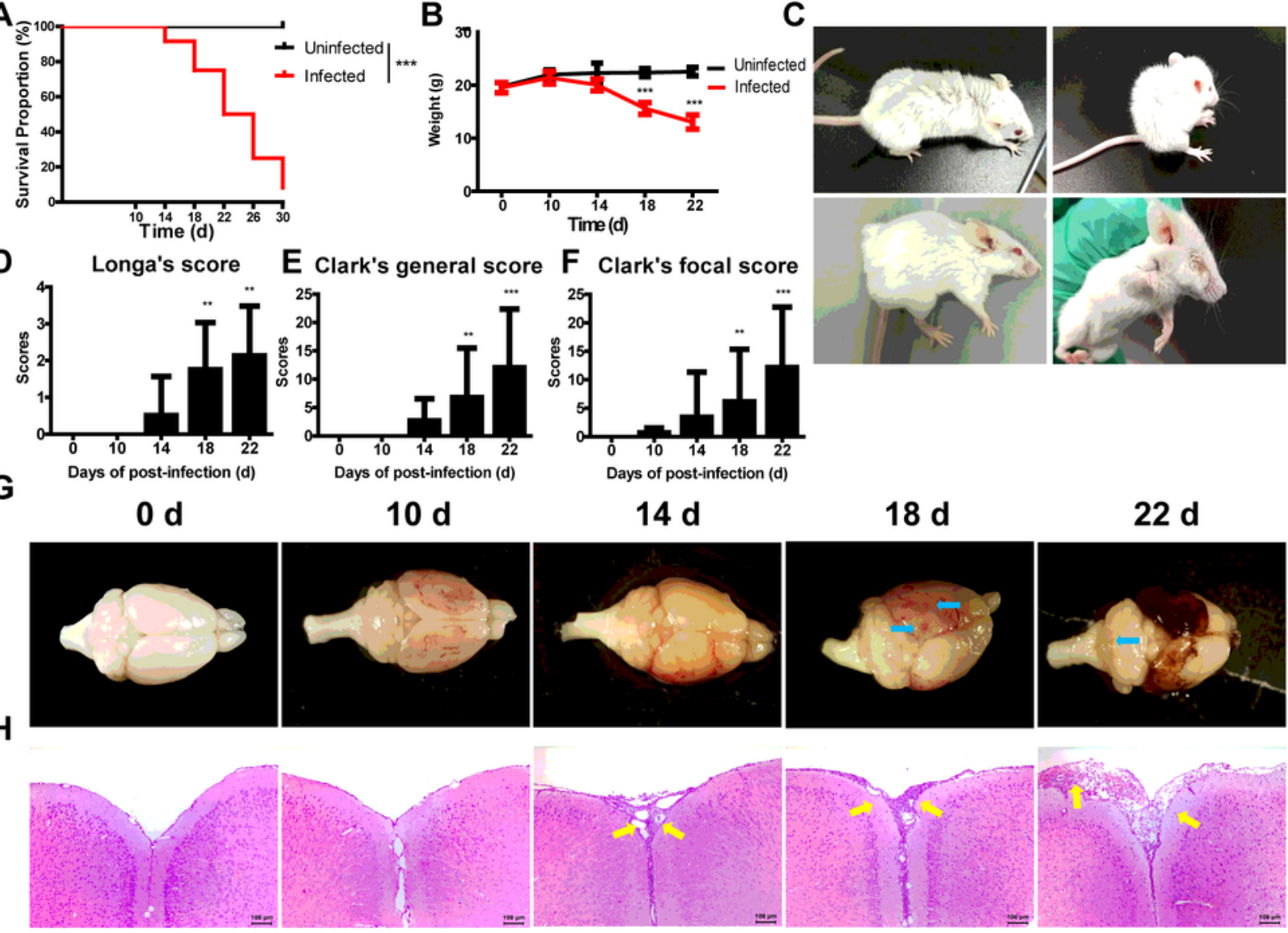

I

IL-1 $\beta$

$\mathbf{J}$

IL-6

$\mathbf{K}$

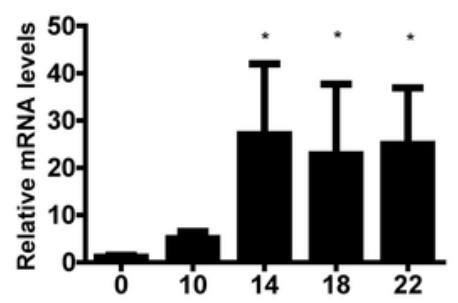

$\mathbf{L}$
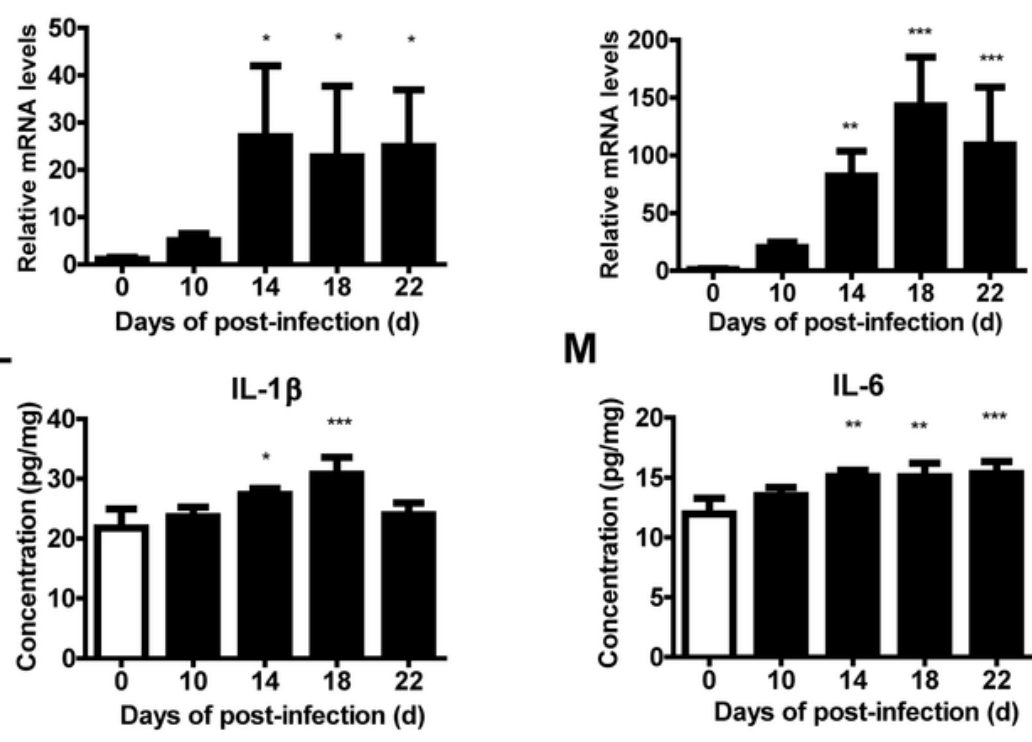

M

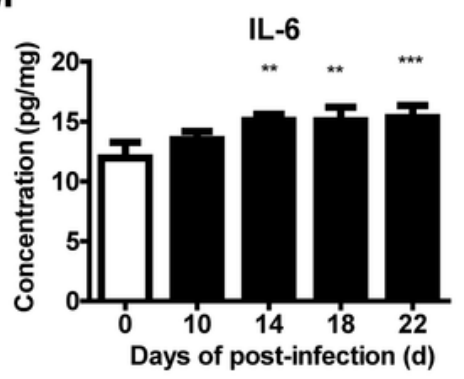

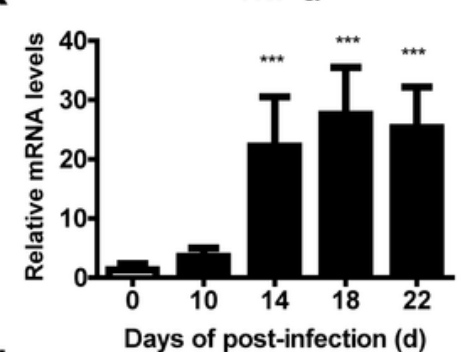

N

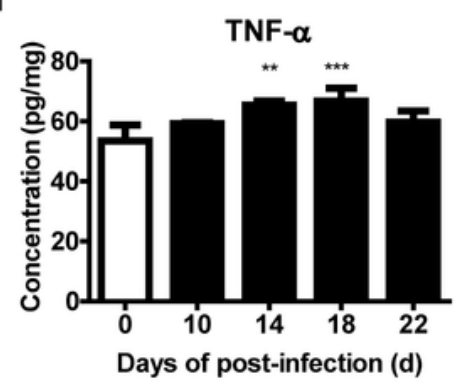

\section{Figure 1}

A. cantonensis infection caused neurological impairment and brain inflammation in mice. Mice were infected with $20 \mathrm{~A}$. cantonensis third-stage larvae by intragastric administration and monitored at 0,10 , 14,18 and $22 \mathrm{dpi}$, respectively. a, b Survival rate and body weight of mice at different infection time points. c Representative images of infected mice with neurologic deficit symptoms. Infected mice displayed various neurologic deficit symptoms including erect hair, arched back, falling to one side when 
walking, blindness and so on. d-f Neurological impairment scores of infected mice. Longa's score, Clark's general score and Clark's focal score were used to evaluate the neurological impairment. g Representative images of brain tissue in infected mice. The brains tissue of infected mice displayed obvious hemorrhage and fourth-stage larvae (blue arrow) from 14 to $22 \mathrm{dpi}$. h Representative histopathological sections of brain tissue in infected mice with H\&E staining. The meninges of infected mice became thick and inflammatory cells infiltrated under the meninges (yellow arrow) from 14 to $22 \mathrm{dpi}$. Images are shown at $100 \times$ magnification (Scale bar, $100 \mu \mathrm{m}$ ). i-n Expression of inflammatory cytokines IL-1 $\beta$, IL- 6 and TNF-a in brain at different infection time points. The gene and protein levels of inflammatory cytokines in the brain of infected mice were detected by qRT-PCR and ELISA. Data are expressed as the means \pm SD. Data showed represent analysis from two independent experiments with four to twelve mice per group.

Survival curve comparison was determined by Log-rank Test. Comparison of body weight was carried by independent-samples $T$ test between infected and uninfected mice. Comparison of the neurological impairment scores was compared by non-parametric test. Multiple comparisons of gene and protein levels of cytokines at different infection time-points were performed using one-way ANOVA. ${ }^{*}<<0.05$; ** $<0.01 ; * \star * P<0.001$ 


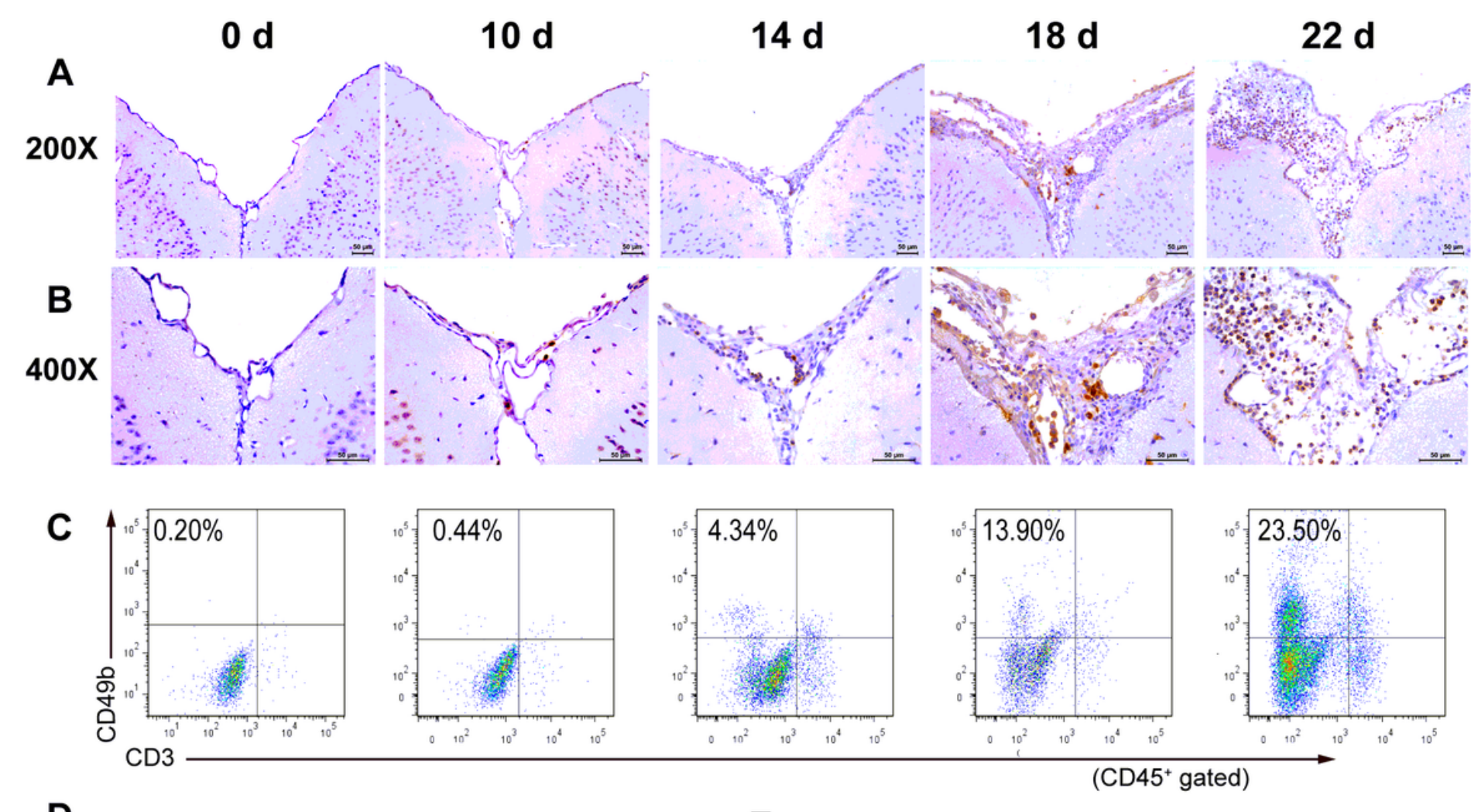

D

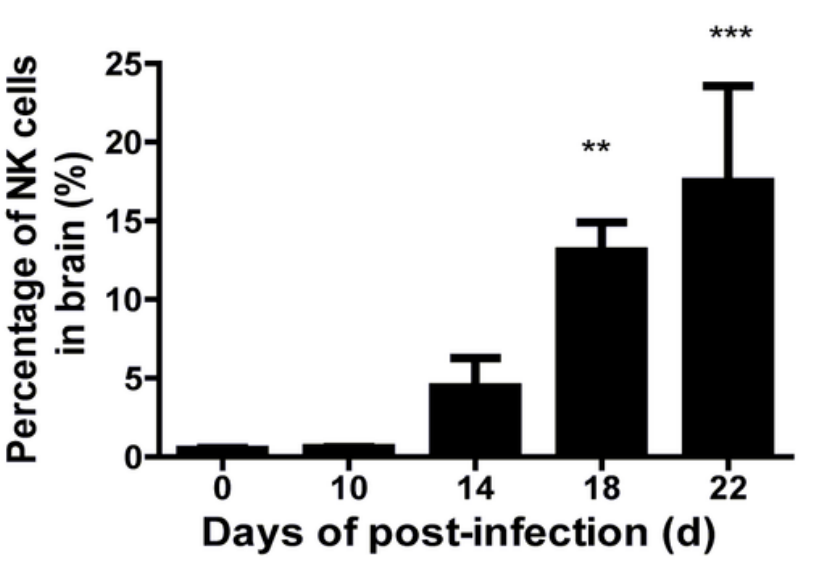

E

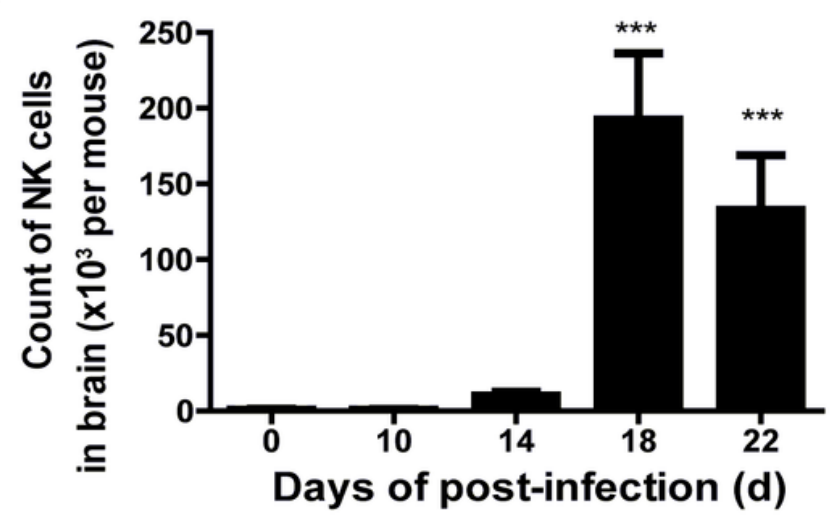

Figure 2

NK cells infiltrated into the CNS after A. cantonensis infection. a, b Representative histopathological sections of brain tissue in infected mice with IHC staining. Brain sections were incubated with rabbit antimouse CD 49b monoclonal antibody and stained with DAB. There were no NK cells in the CNS on $0 \mathrm{dpi}$ and $10 \mathrm{dpi}$. From $14 \mathrm{dpi}$ to $22 \mathrm{dpi}$, more and more NK cells (stained brown) appeared under the meninges. Images are shown at $200 \times$ and $400 \times$ magnification (Scale bar, $100 \mu \mathrm{m}$ ). c-e The percentage and number of NK cells in brain mononuclear cells of infected mice. NK cells in brain mononuclear cells were detected by FCM. The gating strategy for the mouse CD45+CD3-CD49b+ NK cell population in brain was shown in Additional file 2: Figure S2. Data are expressed as the means \pm SD. Data showed represent analysis from two independent experiments with three mice per group. Significance was determined by one-way ANOVA. ${ }^{\star *} \mathrm{P}<0.01 ; * \star \star \mathrm{P}<0.001$ 
od

A
Spleen

Spleen

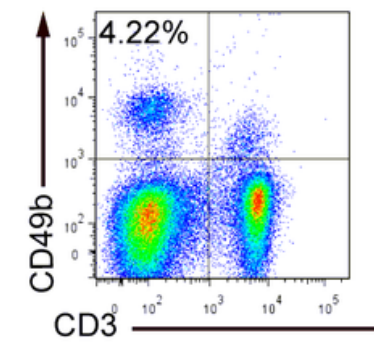

$10 \mathrm{~d}$

$14 \mathrm{~d}$

$18 \mathrm{~d}$

$22 \mathrm{~d}$ CD3

B

B Blood

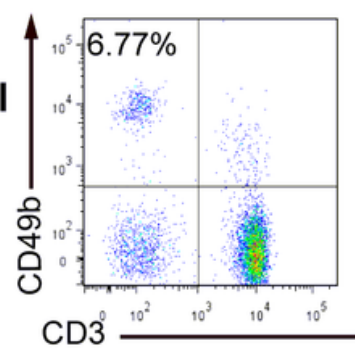

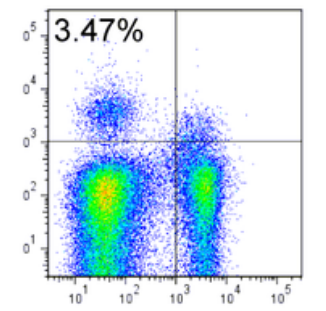

$\begin{array}{lllll}10^{1} & 10^{2} & 10^{3} & 10^{4} & 10^{5}\end{array}$

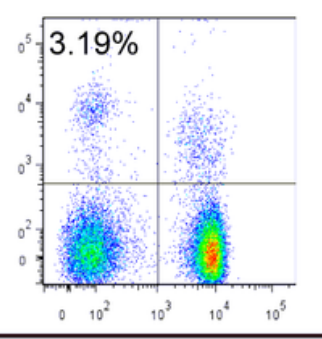

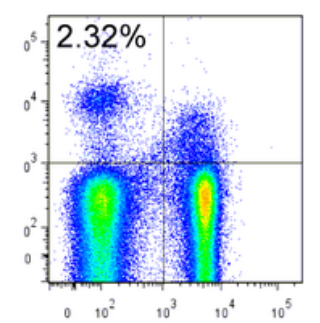

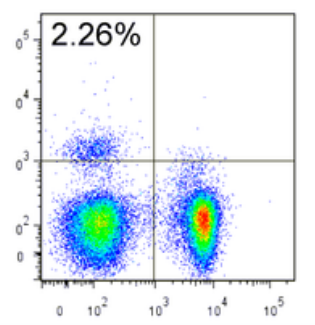

$\left(\mathrm{CD} 45^{+}\right.$gated)
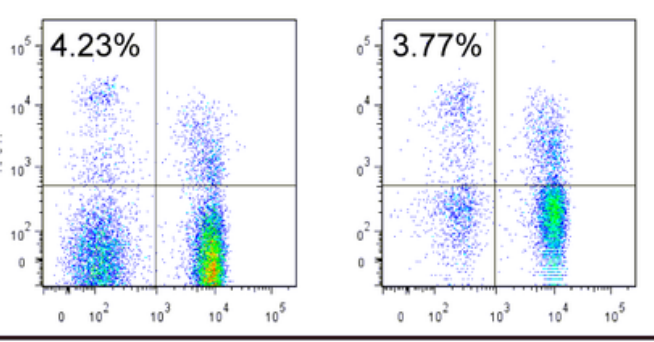
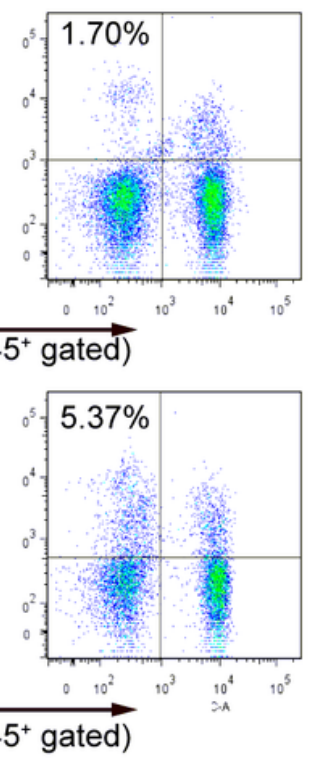

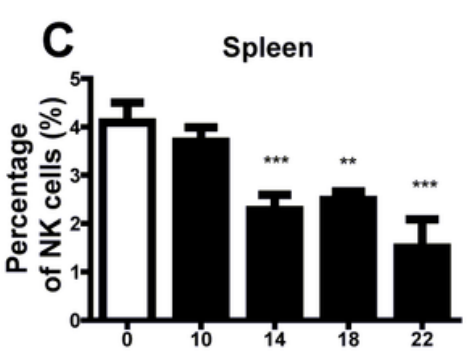

G

Days of post-infection (d)

Correlations between the percentage of sNK and bNK

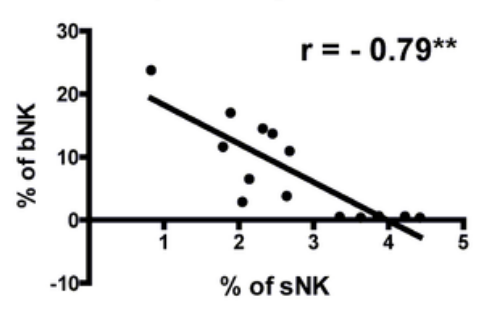

Spleen

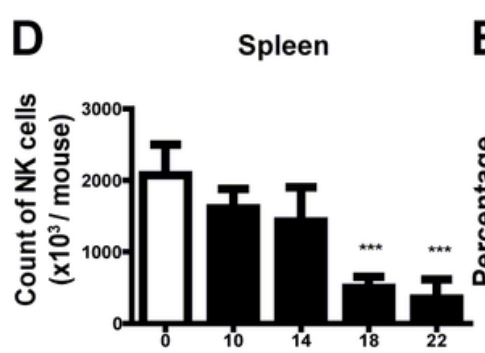

H
E

Peripheral Blood

$\mathbf{F}$

$\left(\mathrm{CD} 45^{+}\right.$gated)

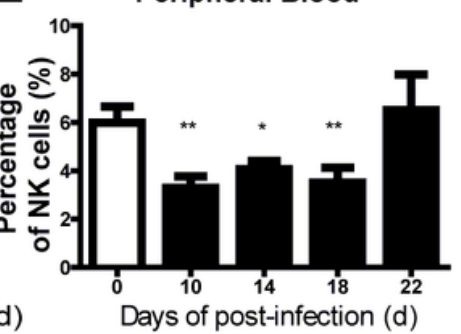

Days of post-infection (d)

Correlations between the count of sNK and bNK

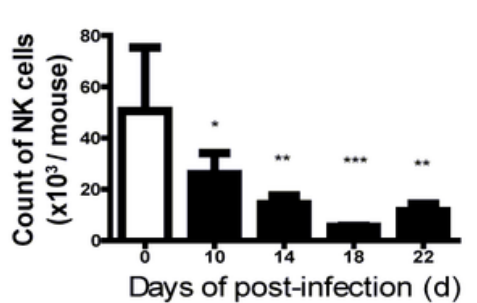

J

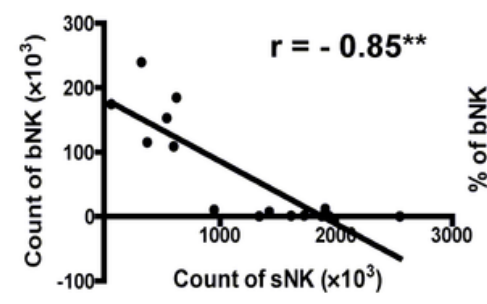

Correlations between the percentage of pbNK and bNK the count of pbNK and bNK

Correlations between

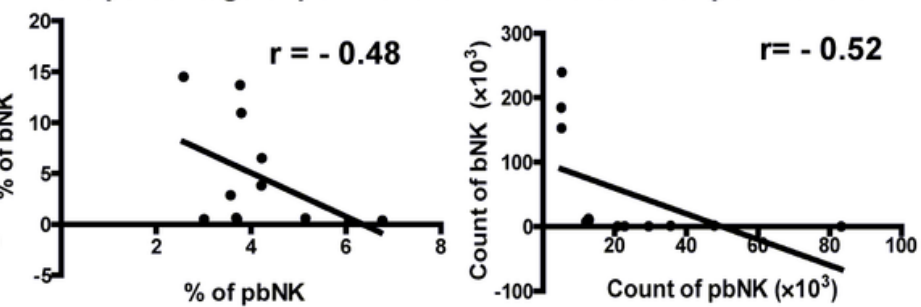

Figure 3

The percentage and number of NK cells in spleen and peripheral blood decreased after A. cantonensis infection. $a, c, d$ The percentage and number of NK cells in splenic lymphocytes of infected mice. $b, e, f$ The percentage and number of NK cells peripheral blood mononuclear cells of infected mice. NK cells in the splenic lymphocytes and peripheral blood mononuclear cells were detected by FCM. $\mathrm{g}, \mathrm{h}$ The correlation between the percentage and number of NK cells in spleen and brain. $\mathrm{i}, \mathrm{j}$ The correlation between the percentage and number of NK cells in peripheral blood and brain. Data are expressed as the means \pm SD. Data showed represent analysis from two independent experiments with three mice per group. Multiple comparisons of the percentage and count of NK cells at different time-points of infection were performed by one-way ANOVA. The correlation between the percentage and number of NK cells in 
different tissues was analyzed by linear correlation. ${ }^{*} \mathrm{P}<0.05$; ${ }^{*} \mathrm{P}<0.01$; ${ }^{* \star} \mathrm{P}<0.001$. sNK, NK cells in spleen; bNK, NK cells in brain; pbNK: NK cells in peripheral blood.
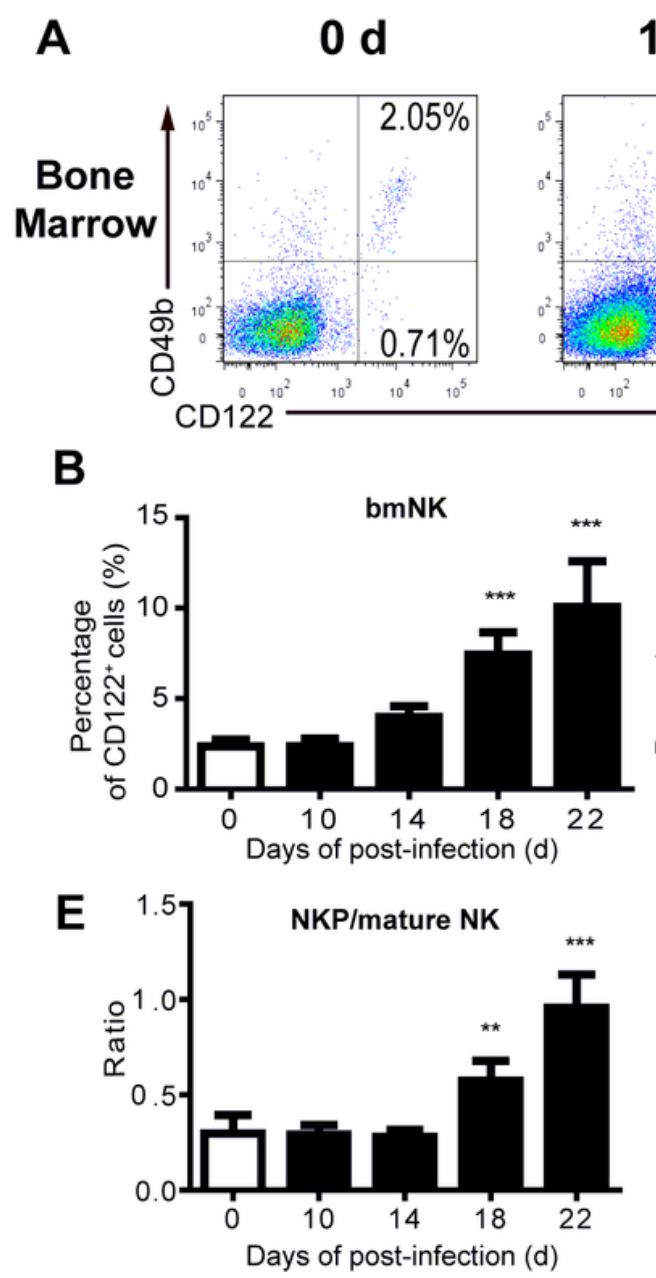

$10 \mathrm{~d}$

$14 \mathrm{~d}$

$18 \mathrm{~d}$

$22 \mathrm{~d}$
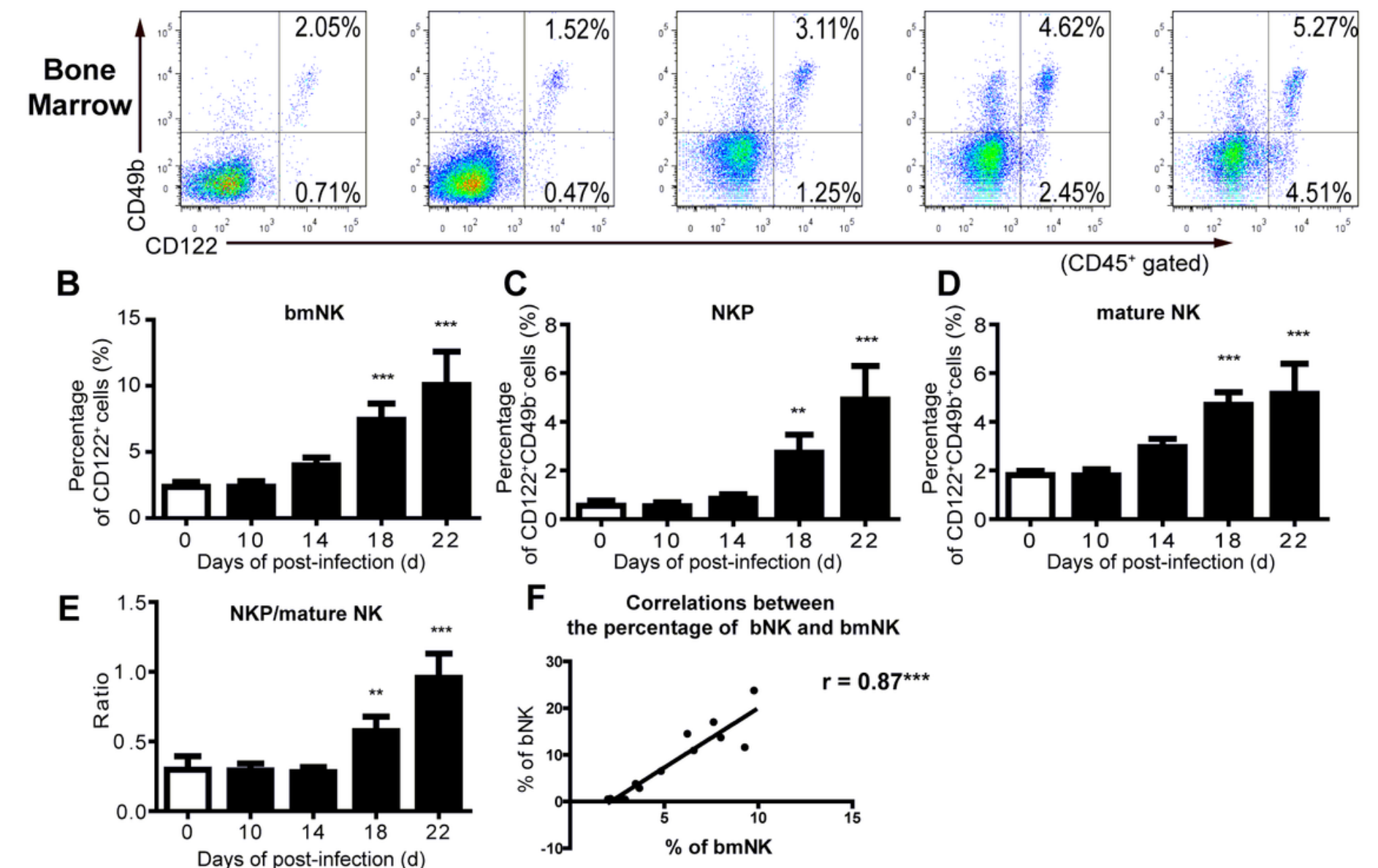

\section{Figure 4}

The percentage of NK cells in bone marrow increased after A. cantonensis infection. a-d The percentage of NK cells in bone marrow lymphocytes of A. cantonensis-infected mice. NK cells in bone marrow (CD122+) were divided into NK precursor cells (CD122+CD49b-) and mature NK cells (CD122+ CD49b+) detected by FCM. e The ratio of NK precursor cells to mature NK cells in bone marrow. $f$ The correlation between the percentage of NK cells in bone marrow and brain. Data are expressed as the means \pm SD. Data showed represent analysis from two independent experiments with three mice per group. Multiple comparisons of the percentage of NK cells at different time-points of infection were performed by oneway ANOVA. The ratio of different time points was analyzed by nonparametric test. The correlation between the percentage and number of NK cells in different tissues was analyzed by linear correlation. ${ }^{\star} * \mathrm{P}<0.01 ;{ }^{* \star *} \mathrm{P}<0.001$. bmNK, NK cells in bone marrow; NKP, NK precursor cells; mature NK cells (mature NK). 
A

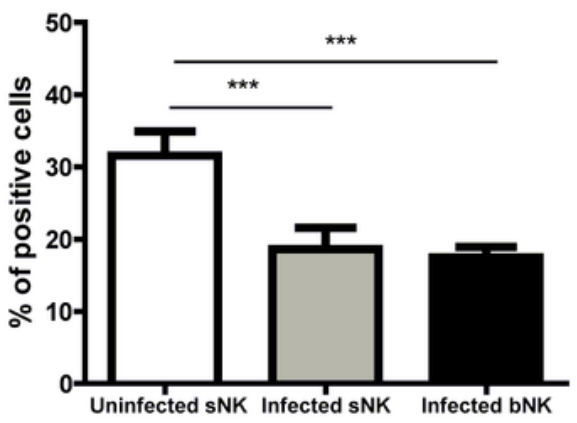

C

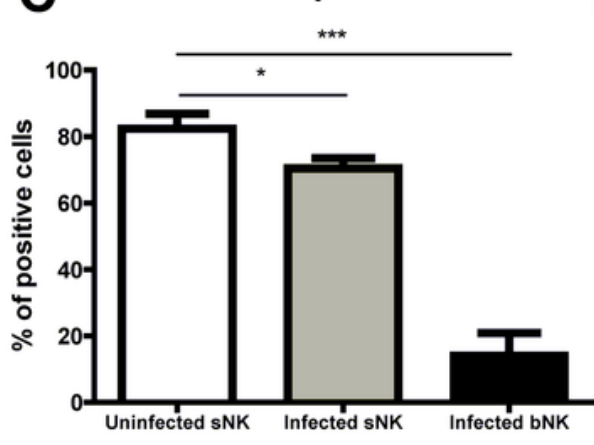

E
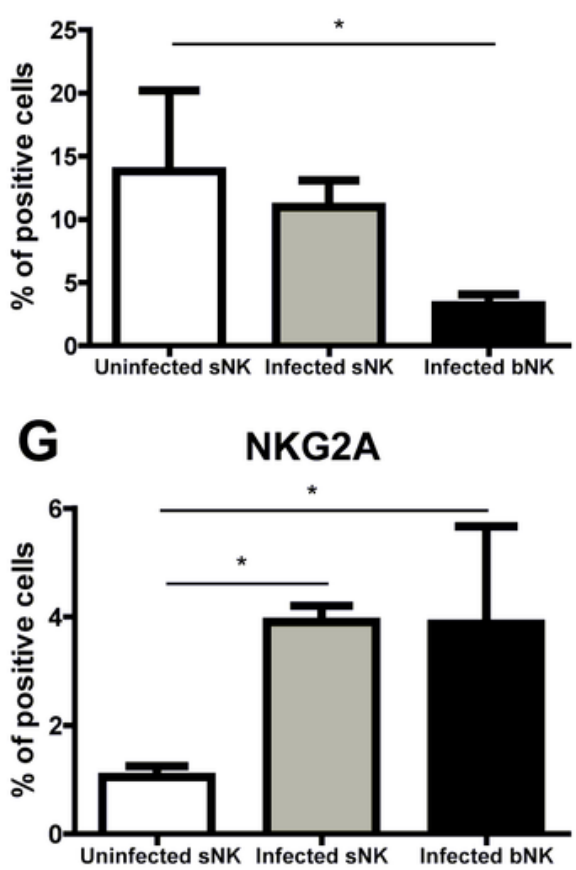
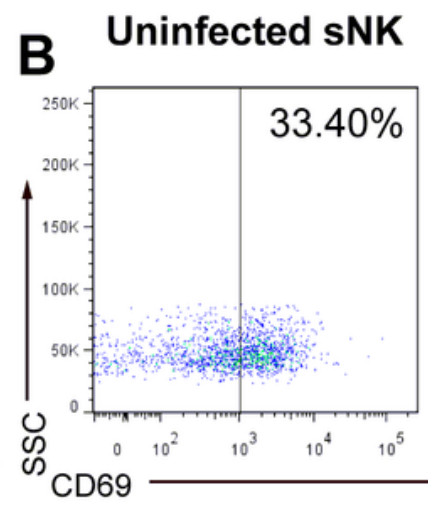

D
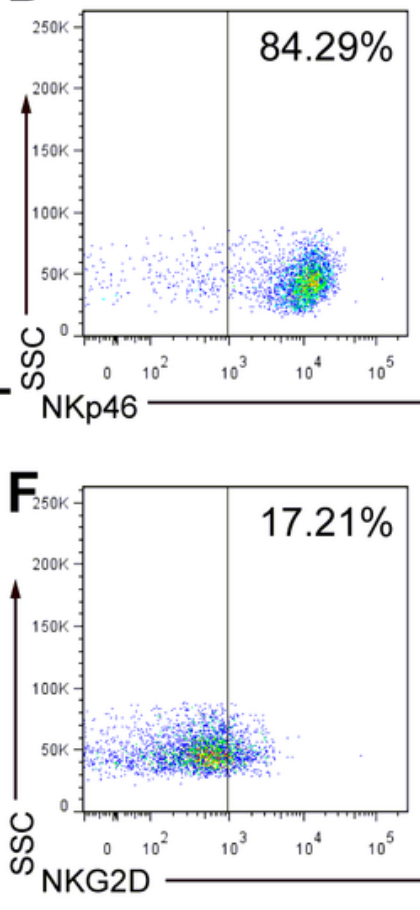

$\mathbf{H}$

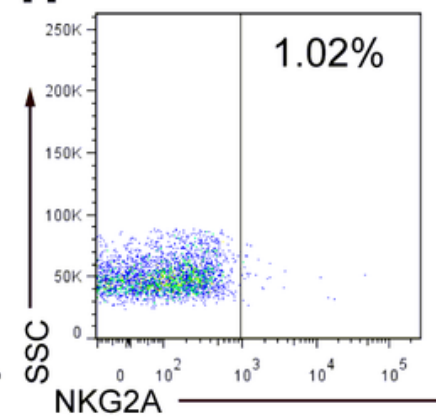

Infected sNK
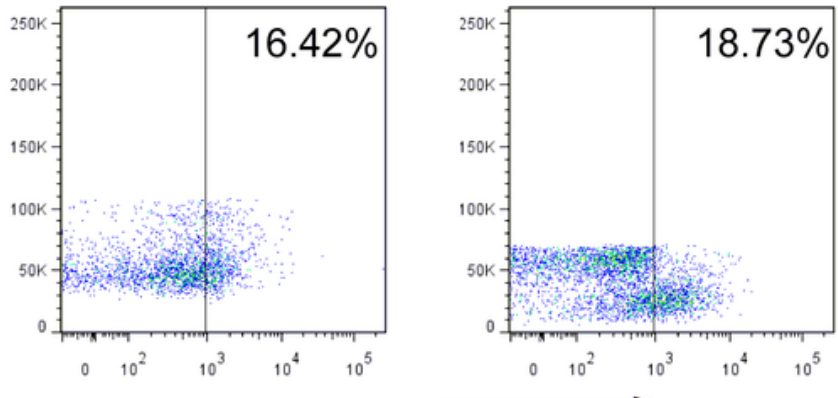

$\left(\mathrm{CD} 45^{+} \mathrm{CD} 3 \cdot \mathrm{CD} 49 \mathrm{~b}^{+}\right.$gated $)$
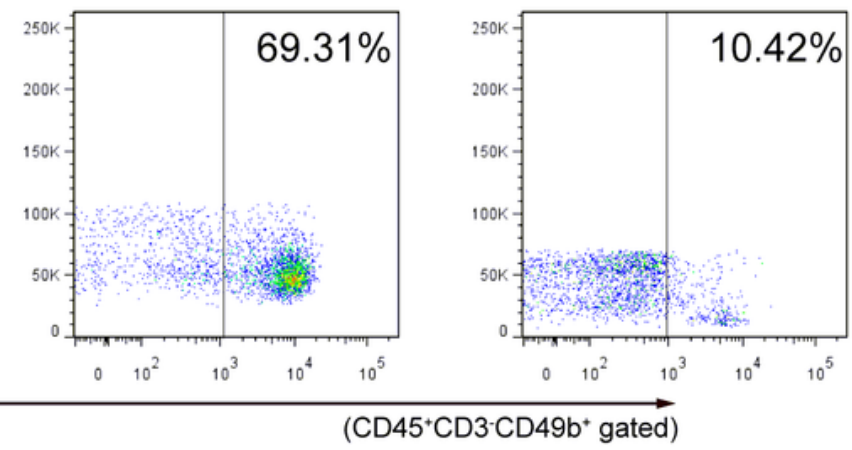

$\left(\mathrm{CD}^{+} 5^{+} \mathrm{CD} 3{ }^{-C D} 49 \mathrm{~b}^{+}\right.$gated $)$

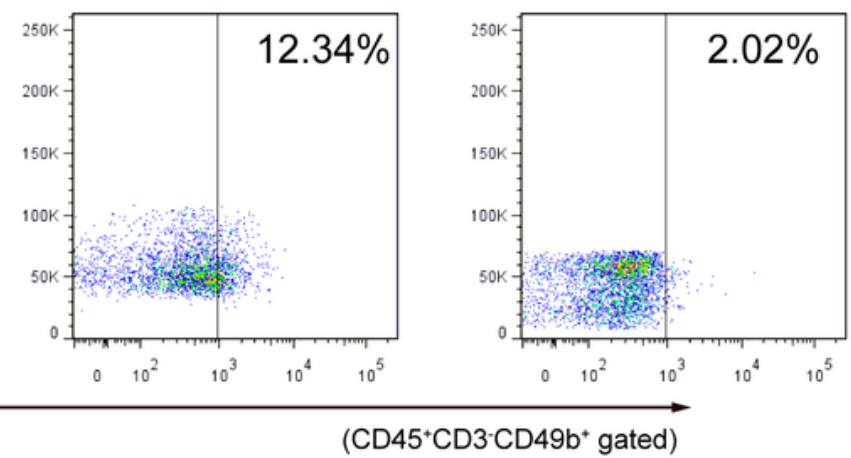

(CD45+CD3 ${ }^{-C D} 49 b^{+}$gated
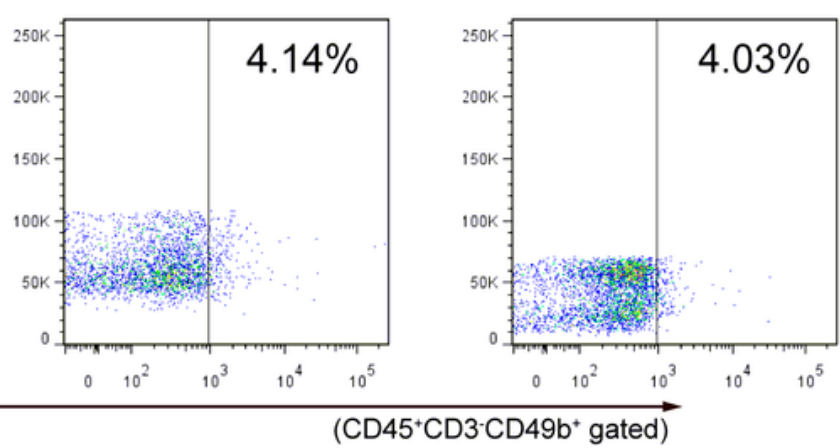

Figure 5

The phenotypes of NK cells changed after A. cantonensis infection. The expression of activation marker CD69 (a, b), activated receptor NKp46 (c, d) and NKG2D (e, f), and inhibitory receptor NKG2A (g, h) on the surface of sNK cells and bNK cells were detected by FCM. Data are expressed as the means \pm SD. Data showed represent analysis from two independent experiments with three mice per group. Multiple 
comparisons of phenotypes between uninfected sNK, infected sNK and infected bNK were performed by ANOVA. ${ }^{*} \mathrm{P}<0.05 ;{ }^{* \star *} \mathrm{P}<0.001$. sNK; NK cells in spleen; $\mathrm{bNK}$, NK cells in brain.
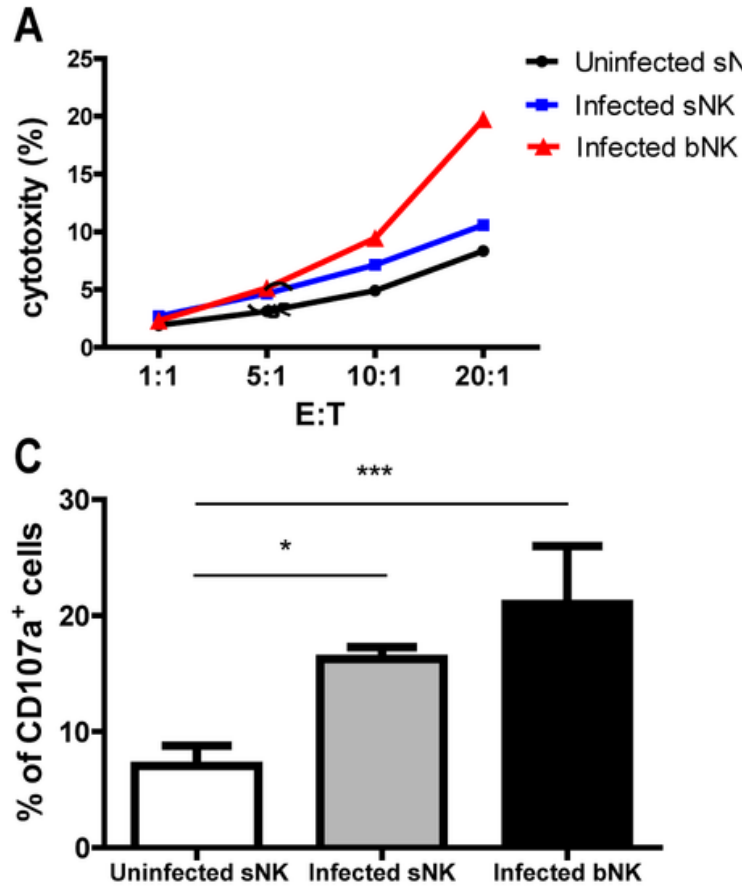

E

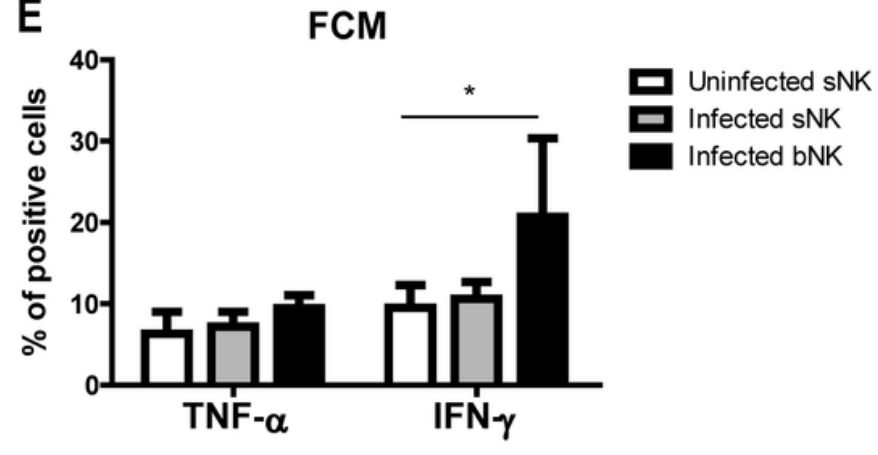

$B$ Uninfected sNK Infected sNK

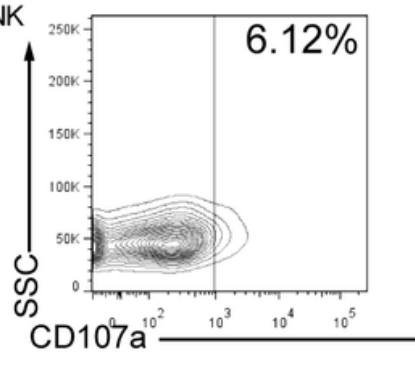

D

ELISA

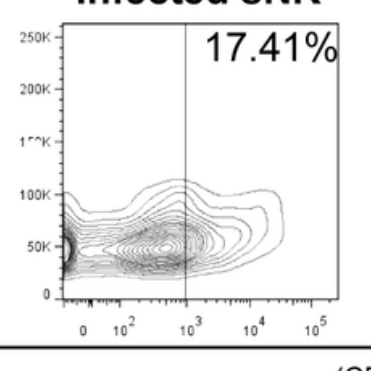

(CD45+CD3-CD49b+ gated)

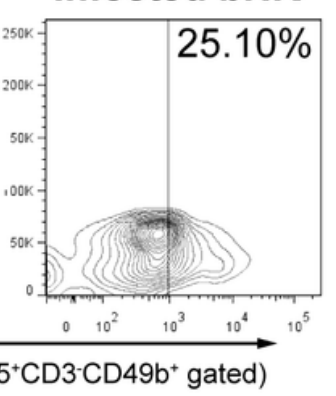
Infected bNK

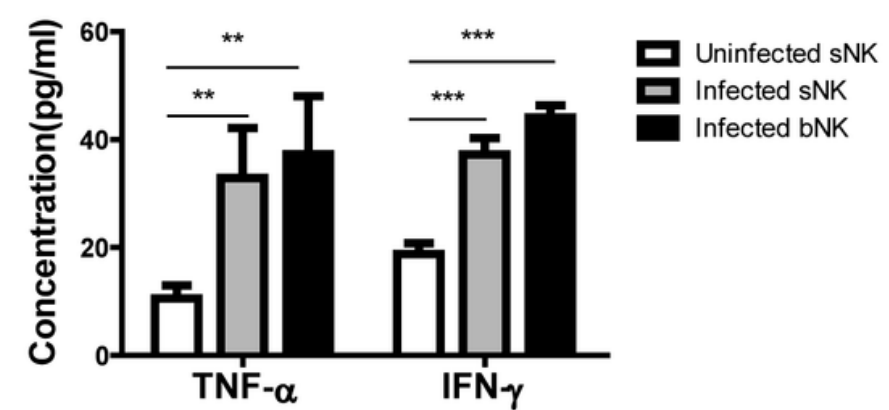

$\mathbf{F}$

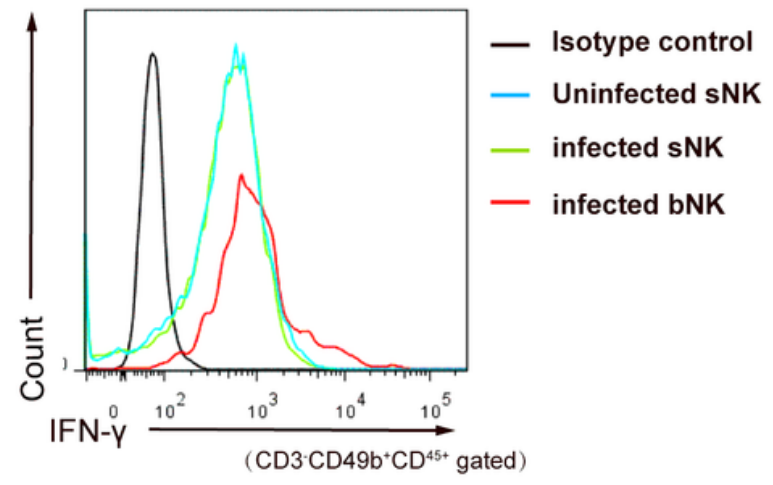

Figure 6

The cytotoxicity and secretory ability of NK cells elevated after A. cantonensis infection. a NK cellmediated cytotoxicity against YAC-1 cells. Purified sNK cells and bNK cells (as effector cells) were incubated with YAC-1 cells (as target cells) at various effector cell/target cell ratios (E:T = 1:1, 5:1, 10:1, 20:1). NK cell-mediated cytotoxicity was detected by LDH release assay and calculated by ODs using the following equation: Cytotoxicity $(\%)=$ (effector/target cell mix - effector cell control - low control) $/$ (high control - low control) $\times 100$. b, c The expression of CD107a on NK cells. Splenic lymphocytes and brain mononuclear cells were isolated and detected by FCM. CD45+CD3-CD49b+ NK cells were gated and analyzed for the expression of CD107a. $d$ The concentrations of TNF- $a$ and IFN- $\gamma$ in the culture supernatants of NK cells. Purified sNKs and bNKs were stimulated with IL-12 and the concentrations of TNF- $a$ and IFN- $y$ in the culture supernatants were detected using ELISA. e, $f$ The expression of intracellular cytokine TNF- $a$ and IFN- $\gamma$ in NK cells. Splenic lymphocytes and brain mononuclear cells were isolated and stimulated with Leukocyte Activation Cocktail. The percentage of TNF-a+ cells and IFN- -+ cells in 

represent analysis from two independent experiments with four mice per group. Multiple comparisons were performed by one-way ANOVA. ${ }^{*} \mathrm{P}<0.05 ;{ }^{\star \star} \mathrm{P}<0.01 ;{ }^{* \star \star} \mathrm{P}<0.001$. sNK; NK cells in spleen; bNK, NK cells in brain.
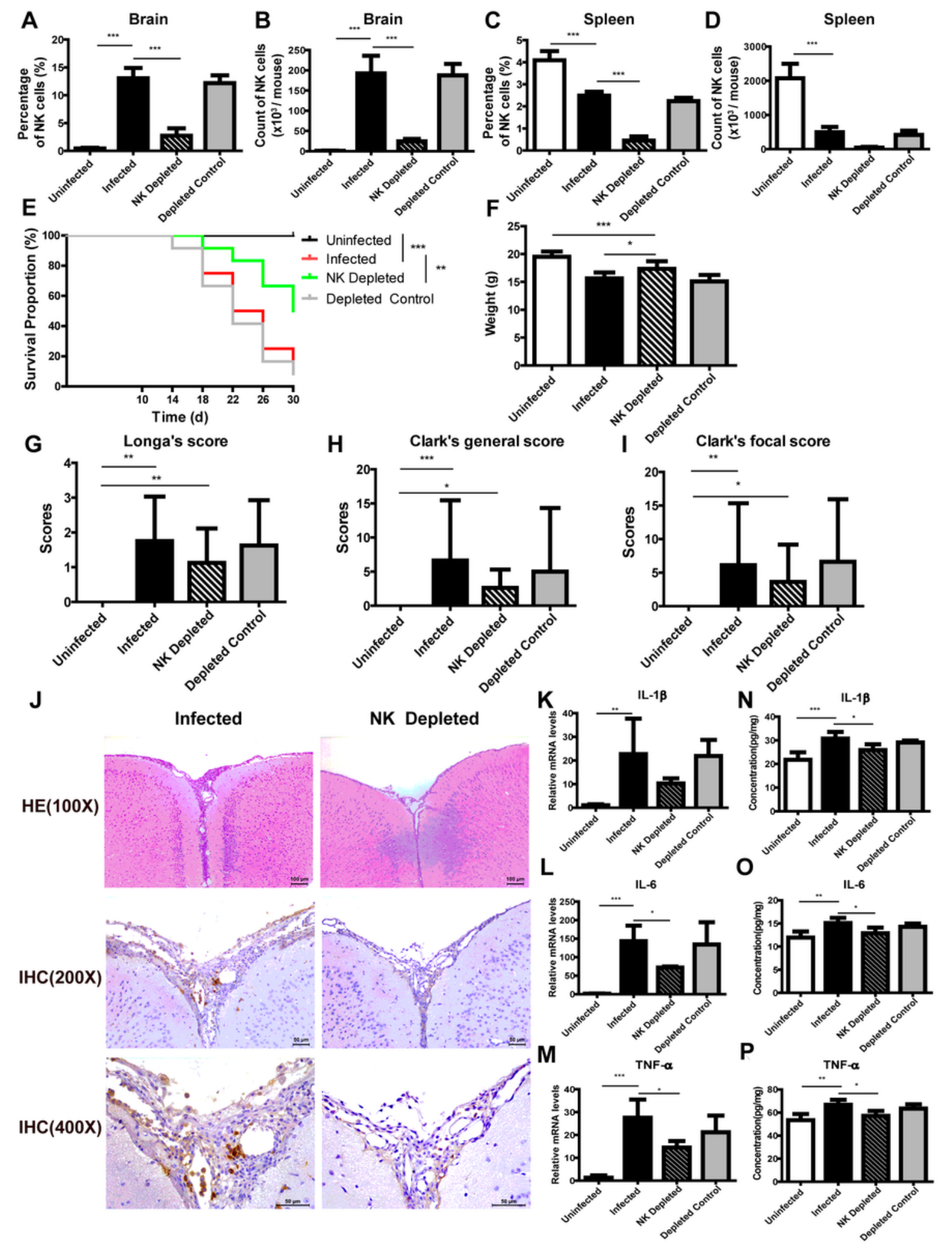

Figure 7 
Depletion of NK cells alleviated brain injury in A. cantonensis-infected mice. Depletion of NK cells in infected mice was induced by tail vein injection of anti-asialo GM1 rabbit serum. The percentage and number of NK cells in brain (a, b) and spleen (c, d) were detected by FCM to evaluate NK cell depleting efficiency. After NK cell depletion, the survival rate of infected mice (e) increased, body weight (f) elevated, while neurological impairment scores ( $\mathrm{g}-\mathrm{i})$ did not have significant changes. Representative histopathological sections of brain tissue with H\&E and IHC staining (j) showed alleviated inflammation and fewer infiltrating NK cells in the brain of NK-depleted mice. Images are shown at $100 \times, 200 \times$ and $400 \times$ magnification (Scale bar, 50-100 $\mu \mathrm{m}$ ). The gene $(k-m)$ and protein levels $(n-p)$ of inflammatory cytokines IL-1 $\beta$, IL- 6 and TNF- $\alpha$ in brain tissue reduced after NK cell depletion measured by qRT-PCR and ELISA. Data are expressed as the means \pm SD. Data showed represent analysis from two independent experiments with three to twelve mice per group. Survival curve comparison was determined by Log-rank Test. Comparison of the neurological impairment scores was compared by non-parametric test. Multiple comparisons of the percentage and number of NK cells, body weight and expression of cytokines were performed using one-way ANOVA. ${ }^{*} \mathrm{P}<0.05 ;{ }^{*} \mathrm{P}<0.01 ;{ }^{* \star *} \mathrm{P}<0.001$. Uninfected, normal mice; Infected, mice infected with A. cantonensis; NK Depleted, infected mice depleted NK cells by injection of anti-asialo GM1 rabbit serum; Depleted Control, infected mice given normal rabbit serum as control. 


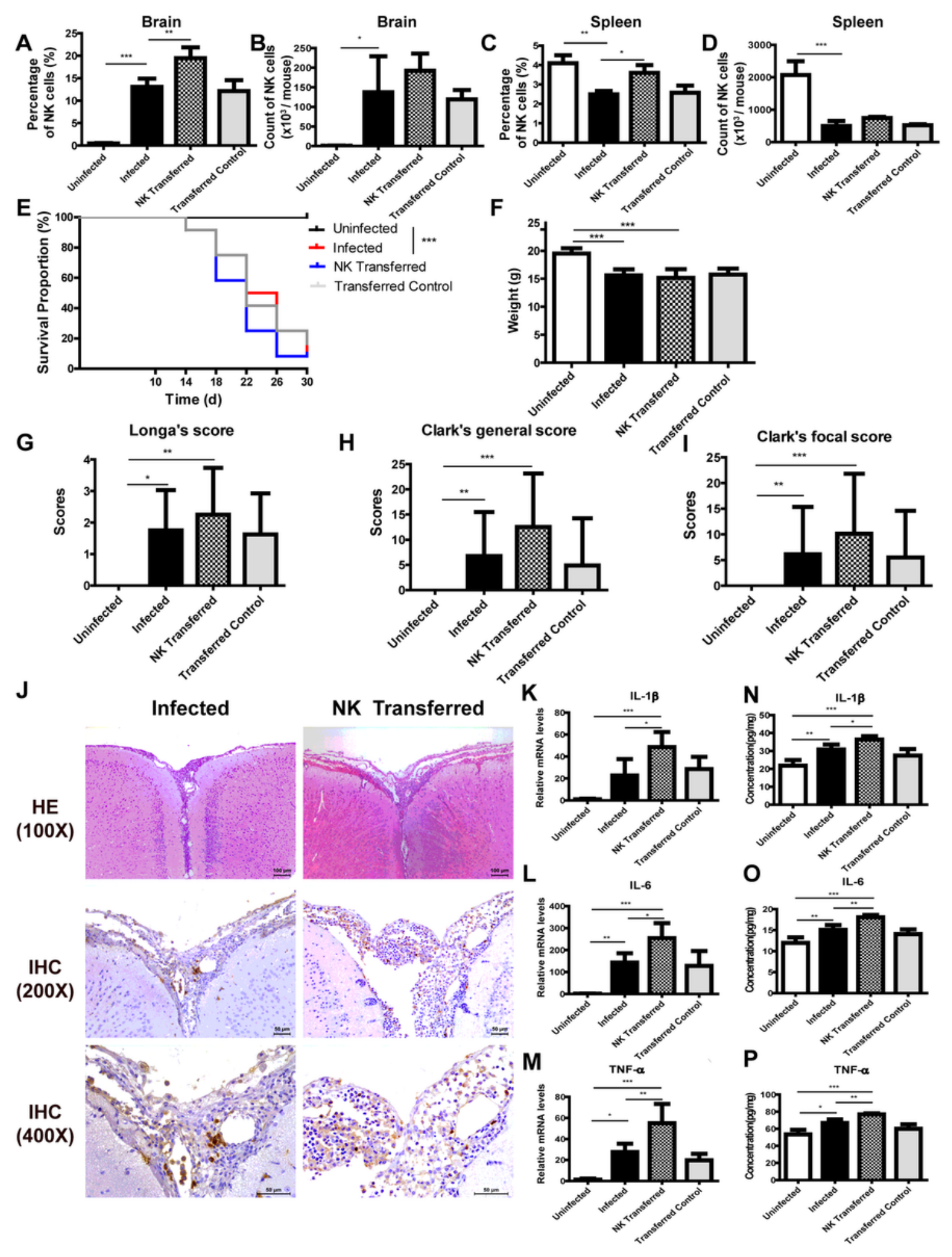

Figure 8

Adoptive transfer of NK cells exacerbated brain damage in A. cantonensis-infected mice. Purified splenic NK cells from normal mice were transferred to infected mice by tail vein injection. The percentage and number of NK cells in brain (a, b) and spleen (c, d) were detected by FCM to evaluate NK cell adoptive transferring efficiency. After adoptive transferring NK cells, the survival rate of infected mice (e), body weight (f) and neurological impairment score ( $\mathrm{g}-\mathrm{i})$ did not change significantly. Representative 
histopathological sections of brain tissue with H\&E and IHC staining (j) showed aggravated inflammation and more infiltrating NK cells in the brain of NK- transferred mice. Images are shown at $100 \times, 200 \times$ and $400 \times$ magnification (Scale bar, 50-100 $\mu \mathrm{m}$ ). The gene $(k-m)$ and protein levels $(n-p)$ of inflammatory cytokines IL-1 $\beta$, IL- 6 and TNF- $\alpha$ in brain tissue elevated after NK cell adoptive transferring measured by qRT-PCR and ELISA. Data are expressed as the means \pm SD. Data showed represent analysis from two independent experiments with three to twelve mice per group. Survival curve comparison was determined by Log-rank Test. Comparison of the neurological impairment scores was compared by non-parametric test. Multiple comparisons of the percentage and number of NK cells, body weight and expression of cytokines were performed using one-way ANOVA. ${ }^{*} \mathrm{P}<0.05 ;{ }^{*} \mathrm{P}<0.01 ;{ }^{* \star *} \mathrm{P}<0.001$. Uninfected, normal mice; Infected, mice infected with A. cantonensis; NK Transferred, infected mice transferred NK cells by tail vein injection; Transferred Control, infected mice injected with PBS as control.

\section{Supplementary Files}

This is a list of supplementary files associated with this preprint. Click to download.

- Additionalfile1FigureS1.tif

- Additionalfile2FigureS2.tif 